\title{
Longitudinal follow-up of health effects among workers handling engineered nanomaterials: a panel study
}

\author{
Wei-Te Wu ${ }^{1,2^{*}}$ (D), Lih-Ann Li ${ }^{1}$, Tsui-Chun Tsou', Shu-Li Wang ${ }^{1}$, Hui-Ling Lee ${ }^{3}$, Tung-Sheng Shih ${ }^{4}$ and \\ Saou-Hsing Liou ${ }^{1,5}$
}

\begin{abstract}
Background: Although no human illness to date is confirmed to be attributed to engineered nanoparticles, occupational epidemiological studies are needed to verify the health effects of nanoparticles. This study used a repeated measures design to explore the potential adverse health effects of workers handling nanomaterials.

Methods: Study population was 206 nanomaterial-handling workers and 108 unexposed controls, who were recruited from 14 nanotechnology plants. They were followed up no less than two times in four years. A questionnaire was used to collect potential confounders and detailed work conditions. Control banding was adopted to categorize risk level for each participant as a surrogate marker of exposure. Health hazard markers include cardiopulmonary dysfunction markers, inflammation and oxidative damage markers, antioxidant enzymes activity, and genotoxicity markers. The Generalized Estimating Equation model was applied to analyze repeated measurements.

Results: In comparison to the controls, a significant dose-dependent increase on risk levels for the change of superoxide dismutase $(p<0.01)$ and a significant increase of glutathione peroxidase change in risk level 1 was found for nanomaterial-handling workers. However, the change of cardiovascular dysfunction, lung damages, inflammation, oxidative damages, neurobehavioral and genotoxic markers were not found to be significantly associated with nanomaterials handling in this panel study.

Conclusions: This repeated measurement study suggests that there was no evidence of potential adverse health effects under the existing workplace exposure levels among nanomaterials handling workers, except for the increase of antioxidant enzymes.
\end{abstract}

Keywords: Nanomaterials, health effect markers, follow-up study, control banding, panel study

\section{Background}

Nanotechnology is regarded as one of the most important industries currently, and the use of nanomaterials in industrial and consumer applications continues to grow. Engineered nanoparticles are now being used in many different types of commercially available products ranging from electronics, to medical and health care products, food, textiles, athletic gear, and household products [1, 2].

\footnotetext{
* Correspondence: ader.una@gmail.com

${ }^{1}$ National Institute of Environmental Health Sciences, National Health

Research Institutes, Miaoli County, Taiwan

${ }^{2}$ Institute of Environmental and Occupational Health Sciences, National Yang

Ming University, Taipei, Taiwan

Full list of author information is available at the end of the article
}

The use of nanomaterials in the development of novel materials for new purposes has led to a rapid increase in the numbers of workers exposed to engineered nanoparticles $[1,2]$. Although nanotechnology has been applied to many different domains, the potential risks generated from its application are often neglected $[2,3]$.

There is a need to assess the risk of potentially adverse health effects among workers handling nanomaterials. Most of the documents about the toxicities of engineered nanoparticles have come mainly from animal or in vitro studies. The health effects induced by engineered nanoparticles in animal inhalation studies include pulmonary fibrosis, granuloma, and inflammation, 
cardiovascular effects, oxidative stress, and pleural plaque formation, lung cancer, and mesothelioma-like effects [2-8]. In addition, needle-like fibrous carbon nanotubes induces an asbestos-like granuloma formation and increases the likelihood of mesothelioma in a tumor prone mouse strain [9]. A recent multi-day, full-shift sampling study among 108 U.S. workers presents unrelated evidence between different metrics of carbon nanotubes and nanofibers and clinically relevant outcomes, included lung function, resting blood pressure, resting heart rate, and complete blood count components or pulmonary symptoms [10]. However, another study in the same research team shows that inhalable Carbon nanotubes and nanofibers structures were associated with matrix metalloproteinase-2 (MMP-2), interleukin-18, glutathione peroxidase (GPx), myeloperoxidase, and superoxide dismutase (SOD) in sputum, and MMP-2, matrix metalloproteinase-9, metalloproteinase inhibitor $1 /$ tissue inhibitor of metalloproteinases 1 , 8-hydroxy-2'-deoxyguanosine, GPx, SOD, endothelin-1, fibrinogen, intercellular adhesion molecule 1 , vascular cell adhesion protein 1, and von Willebrand factor in blood [11]. It implies that the selection of health effect markers (acute or chronic) induced by engineered nanoparticles and using which specimens to test is important.

Most of the evidence for the health effects of engineered nanoparticles on humans was generated from unintentionally produced ultrafine particles. Evidence of human health effects of ultrafine particles (lung inflammation, oxidative damage, worsening of heart disease, atherosclerosis, asthma, and possibly lung cancer) came from air pollution epidemiological studies of unintentionally produced ultrafine particles that were generated from traffic pollution and combustion products, such as diesel exhaust and welding fumes [5, 6]. Epidemiological studies have shown a positive correlation between the particulate matter concentrations in air pollution and increased morbidity and mortality in adults and children $[5,6]$. In addition to healthy individuals, populations having impaired health conditions may become more susceptible to developing health problems from ultrafine particles exposure $[5,6]$.

Although health effects caused by engineered nanoparticles have never been confirmed in humans, there is accumulating evidence from animal studies supporting that exposure to some nanomaterials is harmful. Assessment of risks of engineered nanoparticles exposure requires information on critical target organs and knowledge of toxic endpoints gathered from studies in which relevant exposure routes and exposure levels have been used [12, 13]. Workers involved in the handling of engineered nanomaterials are likely exposed to engineered nanoparticles through inhalation. However, published information on exposures in the workplace is sparse. The main reason for this sparseness is that measuring exposure to engineered nanoparticles is not an easy task $[1,2,13-17]$. The behaviors and characteristics of EN differ in several ways from traditional aerosols [2, 3]. There is still insufficient scientific evidence in regards to the effect of engineered nanoparticles has on health. There is not enough information to determine the range of particle sizes, exposure parameters of the engineered nanoparticles (that should be measured in order to characterize exposure), and which instruments and methods are the most appropriate to use [13-17].

Control banding method has become an acceptable alternative to face with uncertainties relating to nanomaterial exposure [12, 18]. Hazard (severity) bands are generated based on toxicologic data of nanomaterials combined with exposure (probability) bands that reflect the exposure levels. Control banding is a semi-quantitative assessment of risk that offers minimal preventive measures to be implemented according to the estimated levels of risk.

There is no consensus on methodologies for exposure assessment because occupational epidemiological research on engineered nanoparticles is largely lacking. Although we have reported that some adverse health effects may be attributed to engineered nanoparticles exposure [19, 20], longitudinal epidemiological studies still need to be conducted in order to verify the toxic endpoints among workers exposed to engineered nanoparticles. The objective of this exploratory study was to further explore the health effects of workers who handle engineered nanoparticles.

\section{Materials and Methods Study design}

This exploratory study applied a longitudinal design with repeated measurements. This panel study design had five repeated examinations completed within an interval of four years. Nanomaterial-handling workers were recruited from 14 nanomaterials manufacturing and/or using plants in Taiwan. The non-exposed controls were selected from workers who did not handle nanomaterials and who worked at the same plants. This was done in order to have comparable geographic areas and socioeconomic statuses. Blood, urine, and exhaled breath condensate specimens were repeatedly collected at baseline, 6 months later, 18 months later, 30 months later, and 42 months later to measure selected biomarkers after informed consent was obtained from individual participant. Physical examination of pulmonary function, heart rate variability, neurobehavioral test and selfadministered questionnaire were also repeatedly performed. This study has been approved by the institutional review board of the National Health Research Institutes of Taiwan.

\section{Study population}

We have conducted an industrial hygiene survey of the nanotechnology plants in Taiwan [21]. We estimated that about 70 plants were manufacturing and/or using 
nanomaterials in Taiwan. Among them, 39 plants were visited and invited to participate in this epidemiological study. Fourteen plants agreed to participate in this study. Some physicochemical properties and work conditions of nanomaterials collected from 14 plants except for one research institute are listed in Table 1.

We performed five repeat examinations during the four years. A total of 496 workers, including 258 exposed workers and 238 controls, were recruited in this study cohort. Those who have participated in no less than two examinations were included in the data analysis. 314 workers were examined no less than two times, including 206 exposed workers and 108 controls. The response rate of total workers was estimated to be 314/496= $63.3 \%$. The response rate for exposed group was 206/ $258=79.8 \%$, while it was $108 / 238=45.3 \%$ for control group.

\section{Exposure assessment}

Since there is still a lack of consensus on equipment and methodology for personnel sampling of engineered nanoparticles, this study used the control banding nanotool risk level matrix that was proposed by Dr. Paik and his colleagues $[12,18]$ to categorize the risk level of each participant as a surrogate marker of exposure. The risk level matrix was calculated based on the probability scores of the exposure and the severity scores of the nanomaterial toxicity. The variables considered in the exposure probability were collected from individual questionnaire, including the estimated amount of material used (25 points), dustiness/mistiness (30 points), number of employees with similar exposure (15 points), frequency of operation (15 points), and duration of operation (15 points). The factors considered in the calculation of the severity score include nanomaterial $(70 \%$ of severity score) and parent material (30\% of severity score). The factors considered in the calculation of the severity score of the nanomaterials were collected from industrial survey of individual factory, including surface chemistry (10 points), particle shape (10 points), particle diameter (10 points), solubility (10 points), carcinogenicity (6 points), reproductive toxicity (6 points), mutagenicity (6 points), dermal toxicity (6 points), and asthmagenicity (6 points). The factors considered in the calculation of the severity score of parent material include occupational exposure limit (10 points), carcinogenicity (4 points), reproductive toxicity (4 points), mutagenicity (4 points), dermal toxicity (4 points), and asthmagenicity ( 4 points).

In order to obtain consistent scores, the nanomaterial toxicity severity score was based on the toxicity summary tables of a review document [22]. The factors of exposure probability score was based on the questionnaires collected from individual worker exposed to the various nanomaterials. The cross-table of the severity scores (band) and probability scores (band) was used to generate the risk levels (1 to 4 ) for each individual. The higher the risk levels, the higher the risk of health effects.

\section{Health effect markers}

Based on the review of the inhalation studies in humans and animals $[2,4-8,23-28]$, this study investigated six aspects of potential toxic endpoints, including lung inflammation, oxidative damage or lipid peroxidation and antioxidant enzyme activity, cardiovascular diseases markers, DNA damage and genotoxicity, pulmonary function, and neurobehavioral function. Each marker was measured according to standard protocols that were either provided by suppliers or developed by laboratories.

The markers measured for each aspect of health effects include:

1. Inflammation markers, such as Clara cell protein (CC16) [29], heat shock protein 70 [30], nitric oxide (NO) [31, 32], nuclear factor $\mathrm{kB}(\mathrm{NFkB})$ transcription factor activation [33].

2. Oxidative damage markers and antioxidant enzyme activities: such as copper-zinc superoxide dismutase (SOD), glutathione peroxidase-1 (GPX-1) [34, 35], 8-hydroxydeoxyguanosine (8-OHdG) [36, 37], N7 methyl guanosine (N7-MedG) [37], and isoprostane (8-iso-prostaglandin F2 $\alpha$ ) (PGF2 $\alpha$ ) [38].

3. Cardiovascular markers, such as fibrinogen, vascular cell adhesion molecule (VCAM), intercellular adhesion molecule-1 (ICAM-1), interleukin-6 (IL-6), IL-6 soluble receptor (IL6sR) [30, 39], myeloperoxidase (MPO), arylesterase, paraoxonase (PON 1) [40], high-sensitivity C-reactive protein (hsCRP) [41], and heart rate variability (HRV) (including time domain such as standard deviation of all normal to normal R-R intervals (SDNN), root mean square of successive differences between adjacent normal cycles (RMSSD) and frequency domains such as very low frequency (VLF), low frequency (LF), high frequency (HF), ratio of LF to HF (LF/HF)) [42].

4. Genotoxicities using the comet assay, including \%DNA in the tail, tail moment, olive moment, and $\mathrm{L} / \mathrm{H}$ ratio (tail to head ratio) [43], and micronucleus (MN) assay [44].

5. Lung function, including forced vital capacity (FVC), forced expiratory volume at 1 second (FEV1.0), maximal mid-expiratory flow (MMF), peak expiratory flow rate (PEFR), forced expiratory flow at 25\% (FEF25\%), forced expiratory flow at $50 \%$ (FEF50\%), and forced expiratory flow at 75\% (FEF75\%) [45]. 


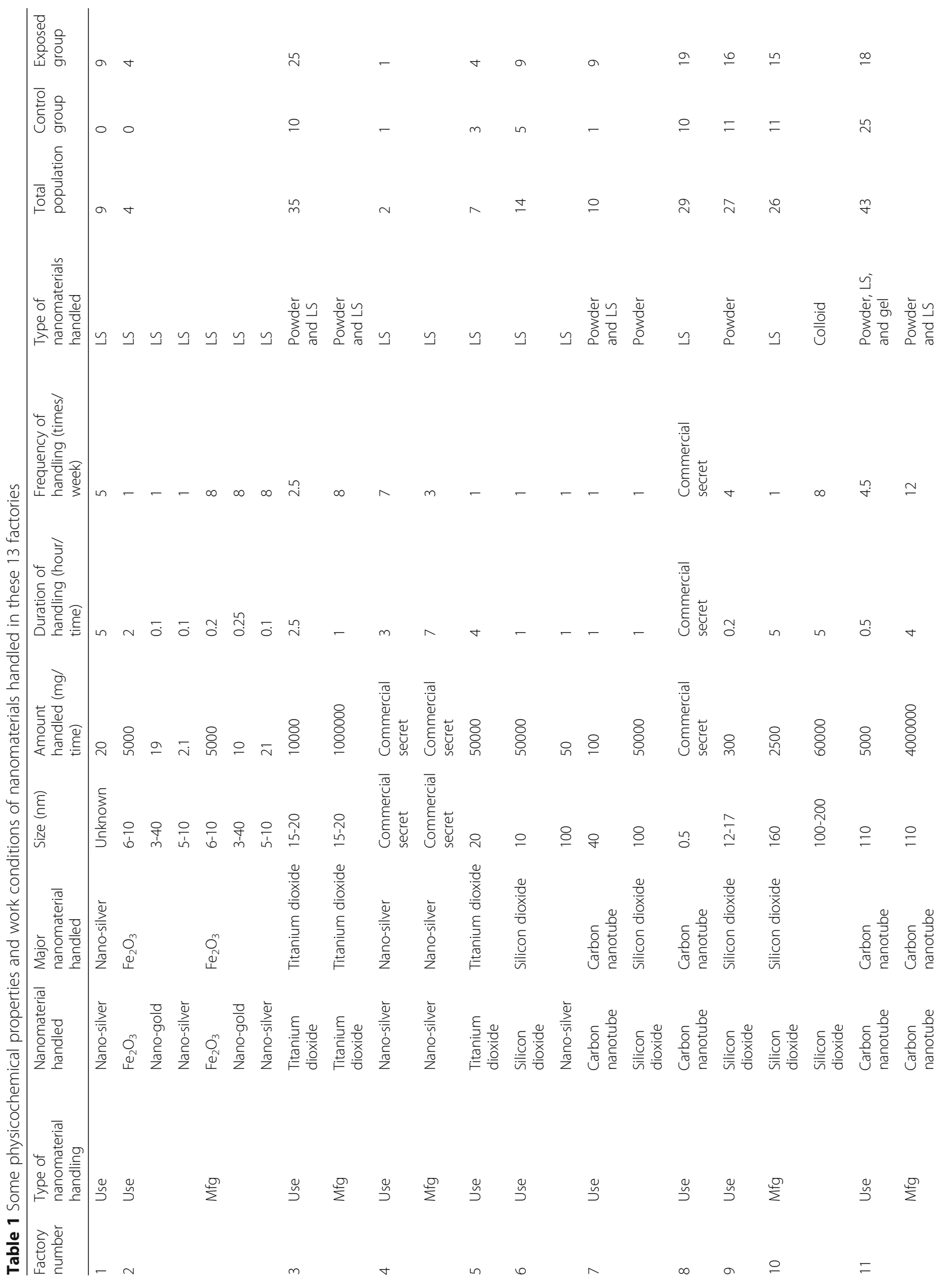




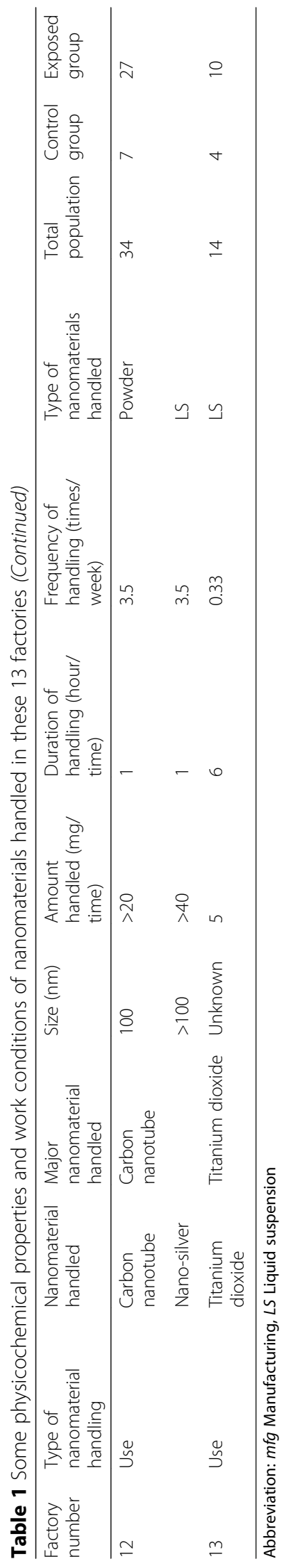


6. Neurobehavioral tests, including reaction time test and 5, 6, 7-digit forward and backward memory tests [46].

\section{Data analysis}

We used SPSS statistical software version 20 (SPSS, Chicago, IL, USA). Means and standard deviations were used to describe the distributions of continuous variables. Percentages were used to describe the distributions of categorical variables. The chi-square test was used to test the differences among the categorical variables. $P<0.05$ was considered statistically significant.

The generalized estimating equation (GEE) model for repeated measurements was used in this data analysis. We have performed five repeated examinations during the four-year. Those who participated in no less than two examinations were included in this data analysis. Two types of analyses were performed. First, the difference in the change of markers among five repeated measurements was analyzed based on the risk level (Risk Level 1*Time vs. control*Time, and Risk Level 2*Time vs. control"Time). Second, the dose-response relationship of change of markers on continues risk levels (Risk Level*Time) was analyzed by trend test. To control for confounding variables, this study first identified potential confounders for each health effect marker. Those variables associated with health effect markers were identified and considered as confounders and adjusted in the GEE model. Since smoking is a strong confounder, it was forced to be adjusted for every effect marker.

In addition to confounders, the study also collected data on potential background exposure to incidental ultrafine particles. It was found that there was no difference among risk level 2, risk level 1, and the control groups regarding frequencies of ultrafine particles exposure resulting from smoking, second-hand smoke, transportation, residential exposure close to traffic roads, residential exposure close to industrial factories (within 50 meters), burning incense in the house, and burning anti-mosquito coils in the house. Therefore, this study did not adjust for incidental ultrafine particles exposure in the final GEE model.

\section{Results}

\section{Physicochemical properties of nanomaterials handled in these 13 factories}

The participating factories include photocatalyst manufacturing, nanomaterials manufacturing, toilet manufacturing, air cleaner manufacturing, light emission device manufacturing, nanopaint manufacturing, colorants manufacturing, carbon nanotube manufacturing, textile manufacturing, and self-cleaning tiles manufacturing factories. These factories were created to handle nanomaterials as early as 2001. The size of nanotechnology factories used in the study was small and the number of employees ranged from 1 to 27. Most factories used local ventilation as a method to control dust emission. Most used water flushing and sweeping to clean the leakage of nanomaterials.

Table 1 shows the physicochemical properties and work condition of nanomaterials in these 13 plants. One factory manufactured nanomaterials only, eight factories used nanomaterials only, and four factories both manufactured and used nanomaterials. The types of nanomaterials handled in these plants include nanosilver, silicon dioxide, titanium dioxide, carbon nanotube, and other nanomaterials. The major types of nanomaterials handled in these factories were silicon dioxide, titanium dioxide, and CNT. The size of nanomaterials handled was usually less than $100 \mathrm{~nm}$.

The amount of nanomaterials handled varied. Some factories handled only a few milligrams, some handled hundreds of grams, and some even handled kilograms. The duration of handling nanomaterials ranged from 0.1 to 7 hours per time. Most of the duration of handling time was less than 1 hour per time. The frequency of handling was usually once per week. Some of factories handled nanomaterials more often than once per week (Table 1).

The physical state of nanomaterials handled was frequently liquid suspension, and powder combined with liquid solutions. Only three factories handled nanomaterial powder. The powder handled included two silicon dioxide powders and one carbon nanotube powder (Table 1).

\section{Distribution of characteristics among study population}

The researchers performed five repeated examinations during four-year follow-up. 134 individuals participated in the first examination, which include 99 exposed workers (53 in risk level 1 (RL1), 43 in risk level 2 (RL2), and 3 in risk level 3 (RL3)) and 35 controls. 225 individuals participated in the second examination, including 153 exposed workers (84 RL1, 64 RL2, and 4 RL3) and 72 controls. 220 individuals participated in the third examination, including 150 exposed workers (76 RL1, 68 RL2, and 6 RL3) and 70 controls. 213 individuals participated in the fourth examination, including 126 exposed workers (61 RL1, 60 RL2, and 5 RL3) and 87 controls. 175 individuals participated in the fifth examination, including 94 exposed workers (48 RL1, 40 RL2, and 6 RL3) and 81 controls. Those who participated in no less than 2 examinations were included in the data analysis. Therefore, the total study population was 314 workers, which included 206 exposed workers and 108 controls who were examined no less than twice.

There were 108 RL1, 93 RL2 and 5 RL3 in the exposed group. This study combined RL3 with RL2 as RL2 in the data analysis. The characteristics of the study participants stratified by risk level are shown in Table 2 . 
Table 2 Demographic distribution in study population

\begin{tabular}{|c|c|c|c|c|c|c|c|}
\hline \multirow[t]{2}{*}{ Variables } & \multicolumn{2}{|c|}{ Control $(n=108)$} & \multicolumn{2}{|c|}{ RL1 $(n=108)$} & \multicolumn{2}{|c|}{ RL2 \& RL3 ( $n=98)$} & \multirow[b]{2}{*}{$p$-value ${ }^{a}$} \\
\hline & $\bar{n}$ & $\%$ & $\bar{n}$ & $\%$ & $\bar{n}$ & $\%$ & \\
\hline \multicolumn{8}{|l|}{ Age } \\
\hline$\leqq 36 y r s$ & 53 & 49.07 & 62 & 57.41 & 57 & 58.16 & \multirow[t]{2}{*}{0.34} \\
\hline$>36 y r s$ & 55 & 50.93 & 46 & 42.59 & 41 & 41.84 & \\
\hline \multicolumn{8}{|l|}{ Gender } \\
\hline Female & 29 & 26.85 & 23 & 21.30 & 18 & 18.37 & \multirow[t]{2}{*}{0.33} \\
\hline Male & 79 & 73.15 & 85 & 78.70 & 80 & 81.63 & \\
\hline \multicolumn{8}{|l|}{ Ethnicity } \\
\hline Taiwanese or South Fujienese & 85 & 78.70 & 89 & 82.41 & 73 & 74.49 & \multirow[t]{3}{*}{0.36} \\
\hline Hakkanese & 16 & 14.81 & 9 & 8.33 & 17 & 17.35 & \\
\hline Others & 7 & 6.48 & 10 & 9.26 & 8 & 8.16 & \\
\hline \multicolumn{8}{|l|}{ Education } \\
\hline Senior high school & 19 & 17.59 & 14 & 12.96 & 20 & 20.41 & \multirow[t]{3}{*}{$<0.01$} \\
\hline College & 62 & 57.41 & 47 & 43.52 & 33 & 33.67 & \\
\hline Institute or higher education & 27 & 25.00 & 47 & 43.52 & 45 & 45.92 & \\
\hline \multicolumn{8}{|l|}{ Smoking } \\
\hline No & 95 & 87.96 & 96 & 88.89 & 77 & 78.57 & \multirow[t]{2}{*}{0.07} \\
\hline Yes & 13 & 12.04 & 12 & 11.11 & 21 & 21.43 & \\
\hline \multicolumn{8}{|l|}{ Alcohol Drinking } \\
\hline No & 101 & 93.52 & 103 & 95.37 & 81 & 82.65 & \multirow[t]{2}{*}{$<0.01$} \\
\hline Yes & 7 & 6.48 & 5 & 4.63 & 17 & 17.35 & \\
\hline \multicolumn{8}{|l|}{ Chewing betel nut } \\
\hline No & 107 & 99.07 & 108 & 100.00 & 93 & 94.90 & \multirow[t]{2}{*}{0.02} \\
\hline Yes & 1 & 0.93 & 0 & 0.00 & 5 & 5.10 & \\
\hline
\end{tabular}

Education, alcohol drinking and betel nut chewing differed significantly by risk levels. Risk level 2 had a higher educational level, more alcohol drinkers and more betel nut chewers, while the control group had more university educated subjects, less alcohol drinkers, and less betel nut chewers. The difference in age, gender, ethnic distribution, and smoking status among the three groups was not significant. The mean length of employment was 8 to 11 years. The mean duration of follow-up was about 2.05 years.

\section{Nanomaterials handled}

The types of nanomaterials handled by the 206 exposed individuals were silicon dioxide, titanium dioxide, carbon nanotubes, nanosilver, and other nanomaterials including nanoresins, nanogold, nanoclay, nanoalumina, and metal oxides. Most frequent type of exposure was mixed types of nanomaterials $(n=121,58.7 \%)$. Silicon dioxide was the most frequent single exposure $(n=88,42.7 \%)$, followed by titanium dioxide $(n=70,34.0 \%)$, carbon nanotubes $(n=68,33.0 \%)$, nanosilver $(n=38,18.4)$, and others $(n=63,30.6 \%)$.

\section{Health effects of nanomaterials handling workers}

There were no significant differences between the exposed and the control workers in changes of lung inflammation markers such as serum Clara cell 16 (CC16), exhaled breath nitric oxide, serum nuclear factor $\mathrm{kB}$ transcription factor activation, and nuclear factor $\mathrm{kB}$ transcription factors activation in exhaled breath condensate (EBC) (Table 3). There were also no significant differences between exposed workers and controls in the changes of oxidative damage or lipid peroxidation markers, such as urine 8-hydroxydeoxyguanosine, urine $\mathrm{N}^{7}$-methyl guanosine, plasma 8-hydroxydeoxyguanosine, and isoprostane (8-iso-prostaglandin $\mathrm{F} 2 \alpha$ ) in exhaled breath condensate (Table 3).

GEE analysis showed that only the change of antioxidant enzyme activity in the exposed group was significantly different from control group (Table 4). The change of superoxide dismutase (SOD) over the five repeated measurements in RL2 of the exposed group were significantly greater than in controls $(p<0.01$, Table 4$)$. In addition, the GEE analysis revealed a significant dosedependent increase on risk levels for the change of SOD 
Table 3 Generalized estimating equation analysis of $\geq 2$ repeated measurements of inflammatory, oxidative damage and lipid peroxidation markers over 4 years

\begin{tabular}{|c|c|c|c|c|c|c|c|c|c|}
\hline \multirow[b]{3}{*}{ Variables $^{\mathrm{a}}$} & \multicolumn{2}{|c|}{ Control } & \multicolumn{2}{|c|}{ Risk Level 1} & \multicolumn{2}{|c|}{ Risk Level 2} & \multicolumn{3}{|c|}{ GEE analysis coefficient $B$ (SE) } \\
\hline & \multirow[b]{2}{*}{$N$} & \multirow[b]{2}{*}{ Mean \pm SD } & \multirow[b]{2}{*}{$N$} & \multirow[b]{2}{*}{ Mean \pm SD } & \multirow[b]{2}{*}{$N$} & \multirow[b]{2}{*}{ Mean \pm SD } & \multicolumn{2}{|c|}{ Model 1} & \multirow{2}{*}{$\frac{\text { Model } 2}{\text { RL*Time }}$} \\
\hline & & & & & & & RL1*Time & RL2*Time & \\
\hline FeNO (ppb) 2nd & 70 & $22.07 \pm 12.94$ & 81 & $18.93 \pm 9.5$ & 66 & $23.59 \pm 14.79$ & $0.16(0.80)$ & $-1.05(0.96)$ & $-0.55(0.48)$ \\
\hline FeNO (ppb) 3rd & 72 & $19.81 \pm 13.09$ & 73 & $15.95 \pm 8.14$ & 69 & $20.33 \pm 11.58$ & & & \\
\hline FeNO (ppb) 4th & 78 & $13.33 \pm 8.31$ & 67 & $12.32 \pm 8.12$ & 65 & $15.88 \pm 11.02$ & & & \\
\hline FeNO (ppb) 5th & 65 & $16.99 \pm 11.56$ & 52 & $17.97 \pm 10.3$ & 55 & $17.1 \pm 11.51$ & & & \\
\hline $\mathrm{CC} 16(\mathrm{ng} / \mathrm{ml}) 1 \mathrm{st}$ & 34 & $5.03 \pm 1.42$ & 50 & $5.77 \pm 1.85$ & 44 & $5.17 \pm 1.54$ & $0.26(0.157)$ & $0.33(0.149) \star$ & $0.17(0.074) \star$ \\
\hline CC16 (ng/ml) 2nd & 73 & $5.7 \pm 2.41$ & 81 & $5.27 \pm 2.06$ & 67 & $5.14 \pm 2.19$ & & & \\
\hline CC16 (ng/ml) 3rd & 73 & $9.01 \pm 2.76$ & 74 & $9.52 \pm 3.08$ & 72 & $9.06 \pm 3.52$ & & & \\
\hline CC16 (ng/ml) 4th & 79 & $6.29 \pm 2.26$ & 68 & $6.39 \pm 2.77$ & 65 & $6.02 \pm 2.71$ & & & \\
\hline CC16 (ng/ml) 5th & 63 & $4.46 \pm 2.61$ & 51 & $4.02 \pm 3.24$ & 56 & $4.18 \pm 2.68$ & & & \\
\hline NF-kB (Serum) (pg/ml) 1st & 33 & $0.53 \pm 0.22$ & 51 & $0.54 \pm 0.2$ & 43 & $0.6 \pm 0.24$ & $0.029(0.026)$ & $0.016(0.028)$ & $0.001(0.014)$ \\
\hline NF-kB (Serum) (pg/ml) 2nd & 73 & $1.09 \pm 0.64$ & 82 & $0.85 \pm 0.52$ & 67 & $0.85 \pm 0.58$ & & & \\
\hline NF-kB (Serum) (pg/ml) 3rd & 67 & $0.73 \pm 0.22$ & 71 & $0.79 \pm 0.27$ & 69 & $0.74 \pm 0.24$ & & & \\
\hline NF-kB (Serum) (pg/ml) 4th & 77 & $0.48 \pm 0.12$ & 66 & $0.5 \pm 0.16$ & 65 & $0.48 \pm 0.14$ & & & \\
\hline NF-kB (Serum) (pg/ml) 5th & 63 & $1.05 \pm 0.4$ & 52 & $1.12 \pm 0.45$ & 57 & $1.03 \pm 0.42$ & & & \\
\hline $\mathrm{NF}-\mathrm{kB}(\mathrm{EBC})(\mathrm{pg} / \mathrm{ml}) 1 \mathrm{st}$ & 33 & $0.9 \pm 0.46$ & 52 & $0.88 \pm 0.46$ & 46 & $0.8 \pm 0.42$ & $-0.045(0.025)$ & $-0.047(0.024)$ & $-0.024(0.012) \star$ \\
\hline $\mathrm{NF}-\mathrm{kB}(\mathrm{EBC})(\mathrm{pg} / \mathrm{ml}) 2 \mathrm{nd}$ & 75 & $0.71 \pm 0.27$ & 81 & $0.63 \pm 0.2$ & 68 & $0.64 \pm 0.24$ & & & \\
\hline $\mathrm{NF}-\mathrm{kB}(\mathrm{EBC})(\mathrm{pg} / \mathrm{ml}) 3 \mathrm{rd}$ & 73 & $0.87 \pm 0.33$ & 74 & $1.03 \pm 0.35$ & 71 & $1.02 \pm 0.34$ & & & \\
\hline NF-kB (EBC) $(\mathrm{pg} / \mathrm{ml}) 4$ th & 77 & $0.56 \pm 0.17$ & 68 & $0.54 \pm 0.15$ & 64 & $0.55 \pm 0.11$ & & & \\
\hline NF-kB (EBC) $(\mathrm{pg} / \mathrm{ml}) 5$ th & 65 & $1.19 \pm 0.44$ & 52 & $1.13 \pm 0.52$ & 57 & $1.08 \pm 0.45$ & & & \\
\hline Urine $8-\mathrm{OHdG}(\mathrm{ng} / \mathrm{mL}) 1 \mathrm{st}$ & 35 & $6.96 \pm 4.09$ & 53 & $7.9 \pm 3.83$ & 45 & $8.68 \pm 4.09$ & $-0.242(0.200)$ & $-0.092(0.210)$ & $-0.06(0.104)$ \\
\hline Urine $8-O H d G(n g / m L)$ 2nd & 69 & $4.78 \pm 3.07$ & 77 & $4.43 \pm 2.76$ & 66 & $4.03 \pm 3.07$ & & & \\
\hline Urine $8-O H d G(n g / m L) 3 r d$ & 71 & $3.48 \pm 2.55$ & 74 & $3.05 \pm 1.89$ & 73 & $3.36 \pm 2.73$ & & & \\
\hline Urine $8-\mathrm{OHdG}(\mathrm{ng} / \mathrm{mL})$ 4th & 78 & $4.96 \pm 4.43$ & 67 & $5.71 \pm 4.3$ & 63 & $6.83 \pm 4.83$ & & & \\
\hline Urine $8-\mathrm{OHdG}(\mathrm{ng} / \mathrm{mL})$ 5th & 66 & $3.85 \pm 2.68$ & 50 & $4.46 \pm 2.72$ & 56 & $4.1 \pm 3.04$ & & & \\
\hline plasma 8-OHdG (pg/mL) 1st & 19 & $18.63 \pm 9.62$ & 31 & $15.11 \pm 8.83$ & 29 & $18.38 \pm 10.67$ & $-0.162(0.810)$ & $-0.596(0.694)$ & $-0.301(0.350)$ \\
\hline plasma 8-OHdG $(\mathrm{pg} / \mathrm{mL})$ 2nd & 73 & $17.26 \pm 13.67$ & 76 & $17.9 \pm 11.99$ & 65 & $20.75 \pm 15.23$ & & & \\
\hline plasma 8-OHdG $(\mathrm{pg} / \mathrm{mL}) 3 \mathrm{rd}$ & 73 & $16.42 \pm 6.7$ & 73 & $16.53 \pm 6.8$ & 70 & $16.96 \pm 6.69$ & & & \\
\hline plasma 8-OHdG $(\mathrm{pg} / \mathrm{mL})$ 4th & 79 & $13.78 \pm 6.04$ & 67 & $14.95 \pm 7.31$ & 64 & $14.51 \pm 6.35$ & & & \\
\hline plasma 8-OHdG $(\mathrm{pg} / \mathrm{mL})$ 5th & 64 & $16.12 \pm 6.86$ & 50 & $15.06 \pm 5.84$ & 57 & $18.77 \pm 7.87$ & & & \\
\hline EBC 8-isoPGF2 $(\mathrm{pg} / \mathrm{mL}) 1 \mathrm{st}$ & 34 & $5.56 \pm 2.01$ & 51 & $5.21 \pm 1.65$ & 46 & $5.25 \pm 1.73$ & $0.565(0.180) \star \star$ & $0.341(0.170) \star$ & $0.179(0.084)^{\star}$ \\
\hline EBC 8-isoPGF2 (pg/mL) 2nd & 72 & $4.15 \pm 2.02$ & 82 & $3.96 \pm 1.61$ & 66 & $4.2 \pm 1.65$ & & & \\
\hline EBC 8-isoPGF2 (pg/mL) 3rd & 72 & $11.36 \pm 6.88$ & 74 & $11.5 \pm 7.55$ & 72 & $12.62 \pm 7.46$ & & & \\
\hline EBC 8-isoPGF2 (pg/mL) 4th & 78 & $4.38 \pm 2.19$ & 67 & $4.58 \pm 2.61$ & 65 & $4.5 \pm 2.35$ & & & \\
\hline EBC 8-isoPGF2 (pg/mL) 5th & 65 & $2.45 \pm 1.76$ & 52 & $2.37 \pm 1.67$ & 57 & $2.52 \pm 1.79$ & & & \\
\hline Urine N7-MedG (ug/mL) 3rd & 71 & $2.23 \pm 1.98$ & 74 & $2.1 \pm 1.81$ & 73 & $2.22 \pm 1.99$ & $0.430(0.408)$ & $0.362(0.457)$ & $0.198(0.225)$ \\
\hline Urine N7-MedG (ug/mL) 4th & 78 & $5.72 \pm 3.67$ & 67 & $7.13 \pm 4.9$ & 64 & $6.43 \pm 4.01$ & & & \\
\hline Urine N7-MedG (ug/mL) 5th & 66 & $5.81 \pm 4.34$ & 52 & $7.42 \pm 4.58$ & 56 & $5.41 \pm 4.23$ & & & \\
\hline
\end{tabular}

atimes of measurements (1st: at baseline; 2 nd: at 6 months later; 3rd: at 18 months later; 4th: at 30 months later; 5 th: at 42 months later)

$\star \star p<0.01 ; \star 0.01<p<0.05$

Analytical Model (Dependent variable: FeNO) includes main effects of sex, smoking, asthma, and rhinitis.

Analytical Model (Dependent variable: CC16) includes main effects of sex, smoking, and chewing betel nut.

Analytical Model (Dependent variable: NF-kB (Serum)) includes main effects of sex, smoking, and education.

Analytical Model (Dependent variable: NF-kB (EBC)) includes main effects of sex, and smoking.

Analytical Model (Dependent variable: Urine 8-OHdG) includes main effects of sex, smoking, valvular heart disease, and folliculitis.

Analytical Model (Dependent variable: plasma 8-OHdG) includes main effects of sex, and smoking.

Analytical Model (Dependent variable: EBC 8-isoPGF2) includes main effects of sex, and smoking.

Analytical Model (Dependent variable: Urine N7-MedG) includes main effects of sex, smoking, chewing betel nut, chronic bronchitis, and pigmentation. 
Table 4 Generalized estimating equation analysis of $\geq 2$ repeated measurements of antioxidant enzymes, DNA damage and reaction time over 4 years

\begin{tabular}{|c|c|c|c|c|c|c|c|c|c|}
\hline \multirow{3}{*}{$\overline{\text { Variables }}^{\text {a. }}$} & \multicolumn{2}{|c|}{ Control } & \multicolumn{2}{|c|}{ Risk Level 1} & \multicolumn{2}{|c|}{ Risk Level 2} & \multicolumn{3}{|c|}{ GEE analysis coefficient B (SE) } \\
\hline & \multirow[b]{2}{*}{ N } & \multirow[b]{2}{*}{ Mean \pm SD } & \multirow[b]{2}{*}{ N } & \multirow[b]{2}{*}{ Mean \pm SD } & \multirow[b]{2}{*}{$N$} & \multirow[b]{2}{*}{ Mean \pm SD } & \multicolumn{2}{|c|}{ Model 1} & \multirow{2}{*}{$\begin{array}{l}\text { Model } 2 \\
\text { RL1*Time }\end{array}$} \\
\hline & & & & & & & RL1*Time & RL2*Time & \\
\hline $\mathrm{SOD}(\mathrm{U} / \mathrm{ml}) 1 \mathrm{st}$ & 34 & $7.98 \pm 2.17$ & 53 & $8.61 \pm 3.55$ & 45 & $8.11 \pm 3.5$ & $0.487(0.278)$ & $0.720(0.278) \star \star$ & $0.355(0.136) \star \star$ \\
\hline $\mathrm{SOD}(\mathrm{U} / \mathrm{ml}) 2 \mathrm{nd}$ & 74 & $9.48 \pm 5.04$ & 82 & $7.67 \pm 5.1$ & 68 & $7.79 \pm 5.96$ & & & \\
\hline $\mathrm{SOD}(\mathrm{U} / \mathrm{ml}) 3 \mathrm{rd}$ & 73 & $16.86 \pm 7.4$ & 74 & $19.57 \pm 8.77$ & 72 & $20.3 \pm 9.04$ & & & \\
\hline $\mathrm{SOD}(\mathrm{U} / \mathrm{ml}) 4 \mathrm{th}$ & 78 & $12.67 \pm 3.63$ & 67 & $12.1 \pm 2.76$ & 65 & $11.87 \pm 2.9$ & & & \\
\hline $\mathrm{SOD}(\mathrm{U} / \mathrm{ml}) 5$ th & 64 & $13.38 \pm 4.68$ & 52 & $11.88 \pm 4.31$ & 57 & $13.43 \pm 4.98$ & & & \\
\hline GPx (nmol/min/ml) 1st & 35 & $117.07 \pm 20.67$ & 53 & $120.44 \pm 16.18$ & 44 & $128.02 \pm 29.2$ & $3.652(1.319) \star \star$ & $1.612(1.470)$ & $0.759(0.724)$ \\
\hline GPx (nmol/min/ml) 2nd & 74 & $95.52 \pm 26.18$ & 82 & $80.47 \pm 24.87$ & 68 & $82.97 \pm 25.18$ & & & \\
\hline GPx $(\mathrm{nmol} / \mathrm{min} / \mathrm{ml}) 3 \mathrm{rd}$ & 73 & $157.11 \pm 24.04$ & 74 & $153.8 \pm 21.9$ & 72 & $151.17 \pm 29.02$ & & & \\
\hline GPx $(\mathrm{nmol} / \mathrm{min} / \mathrm{ml})$ 4th & 79 & $100.09 \pm 14.67$ & 68 & $99.32 \pm 15.35$ & 65 & $104.41 \pm 14.38$ & & & \\
\hline GPx $(\mathrm{nmol} / \mathrm{min} / \mathrm{ml}) 5$ th & 64 & $129.49 \pm 19.49$ & 52 & $124.34 \pm 16.85$ & 57 & $130.91 \pm 16.4$ & & & \\
\hline$\%$ DNA in Tail 1st & 35 & $13.43 \pm 11.74$ & 53 & $16.69 \pm 9.84$ & 46 & $14.82 \pm 8.34$ & $2.280(0.792) \star \star$ & $2.240(0.794) \star \star$ & $1.075(0.379) \star \star$ \\
\hline$\%$ DNA in Tail 2nd & 75 & $33.98 \pm 21.37$ & 82 & $22.47 \pm 15.01$ & 68 & $21.69 \pm 20.91$ & & & \\
\hline \%DNA in Tail 3rd & 73 & $34.68 \pm 22.19$ & 74 & $26.83 \pm 20.59$ & 72 & $32.28 \pm 21.77$ & & & \\
\hline$\%$ DNA in Tail 4th & 79 & $18.37 \pm 8.36$ & 68 & $17.69 \pm 6.53$ & 65 & $16.4 \pm 7.91$ & & & \\
\hline$\%$ DNA in Tail 5th & 63 & $16.06 \pm 5.27$ & 52 & $18.01 \pm 6.19$ & 57 & $15.59 \pm 5.25$ & & & \\
\hline Tail Moment 1st & 35 & $17.3 \pm 23.2$ & 53 & $15.16 \pm 14.95$ & 46 & $15.99 \pm 16.21$ & $16.184(3.802) \star \star$ & $14.495(3.970) \star \star$ & $7.052(1.790) \star \star$ \\
\hline Tail Moment 2nd & 65 & $112.23 \pm 106.89$ & 78 & $48.75 \pm 45.96$ & 61 & $37.22 \pm 68.26$ & & & \\
\hline Tail Moment 3rd & 73 & $145.68 \pm 129.55$ & 74 & $106.66 \pm 122.55$ & 72 & $143.41 \pm 135.74$ & & & \\
\hline Tail Moment 4th & 79 & $35.16 \pm 35.07$ & 68 & $29.16 \pm 26.23$ & 65 & $26.93 \pm 29.46$ & & & \\
\hline Tail Moment 5th & 63 & $28.67 \pm 18.75$ & 51 & $30.09 \pm 18.03$ & 57 & $25.11 \pm 19.39$ & & & \\
\hline Olive Moment 1st & 35 & $8.92 \pm 10.45$ & 53 & $8.8 \pm 7.37$ & 46 & $8.42 \pm 6.8$ & $6.188(1.698) \star \star$ & $6.302(1.678) \star \star$ & $2.990(0.768) \star \star$ \\
\hline Olive Moment 2nd & 61 & $43.81 \pm 42.99$ & 77 & $21.71 \pm 15.58$ & 59 & $12.96 \pm 12.98$ & & & \\
\hline Olive Moment 3rd & 73 & $77.18 \pm 73.51$ & 74 & $53.61 \pm 67.1$ & 72 & $73.93 \pm 75.04$ & & & \\
\hline Olive Moment 4th & 79 & $16.92 \pm 14.56$ & 68 & $14.57 \pm 11.31$ & 65 & $13.17 \pm 12.17$ & & & \\
\hline Olive Moment 5th & 63 & $13.77 \pm 7.11$ & 51 & $14.65 \pm 7.64$ & 57 & $12.93 \pm 9.18$ & & & \\
\hline $\mathrm{L} / \mathrm{H}$ ratio $1 \mathrm{st}$ & 35 & $0.99 \pm 0.92$ & 53 & $0.96 \pm 0.61$ & 46 & $0.99 \pm 0.73$ & $0.006(0.036)$ & $0.025(0.035)$ & $0.012(0.018)$ \\
\hline $\mathrm{L} / \mathrm{H}$ ratio 2 nd & 74 & $1.08 \pm 0.87$ & 81 & $1.02 \pm 0.9$ & 68 & $0.81 \pm 0.86$ & & & \\
\hline $\mathrm{L} / \mathrm{H}$ ratio $3 \mathrm{rd}$ & 71 & $0.7 \pm 0.67$ & 73 & $0.51 \pm 0.63$ & 71 & $0.66 \pm 0.7$ & & & \\
\hline $\mathrm{L} / \mathrm{H}$ ratio 4 th & 77 & $0.23 \pm 0.12$ & 68 & $0.22 \pm 0.1$ & 65 & $0.21 \pm 0.13$ & & & \\
\hline L/H ratio 5th & 63 & $0.2 \pm 0.08$ & 50 & $0.21 \pm 0.08$ & 57 & $0.19 \pm 0.08$ & & & \\
\hline
\end{tabular}

a times of measurements (1st: at baseline; 2 nd: at 6 months later; 3rd: at 18 months later; 4th: at 30 months later; 5 th: at 42 months later)

$\star \star p$-values interaction term $<0.01$ in the GEE model

Analytical Model (Dependent variable: SOD) includes main effects of age, sex, smoking, chewing betel nut, and education.

Analytical Model (Dependent variable: GPx) includes main effects of sex, smoking, drinking, hypertension, and atopic dermatitis.

Analytical Model (Dependent variable: \%DNA in Tail) includes main effects of age, sex, smoking, and education.

Analytical Model (Dependent variable: Tail Moment) includes main effects of sex, and smoking.

Analytical Model (Dependent variable: Olive Moment) includes main effects of sex, and smoking.

Analytical Model (Dependent variable: L/H ratio) includes main effects of sex, smoking, and atopic dermatitis.

$(p<0.01)$. The increase in the change of glutathione peroxidase-1 (GPx) over the five repeated measurements in RL1 of the exposed group were significantly greater than in controls $(p<0.01$, Table 4$)$, but were not significant for RL2 and trend test.
There were no significant differences between the exposed and control workers in the changes of genotoxicity markers such as the comet assays and for the change of neurobehavioral test such as reaction time (Table 4). 
No significant differences between the exposed and control workers were found for the changes of cardiovascular dysfunction markers, including fibrinogen, VCAM, ICAM, IL- 6, IL- 6sR, paraoxonase, arylesterase, myeloperoxidase, high-sensitivity C-reactive protein, and heart rate variability parameters including SDNN, RMSSD, VLF, LF, HF, and LF/HF (Table 5, 6 and 7).

There were also no significant differences between the exposed and control workers for the changes of lung function test (Table 8 and 9), including forced vital capacity, forced expiratory volume at 1 second.0, forced expiratory flow at $25 \%$, forced expiratory flow at $50 \%$, and forced expiratory flow at $75 \%$.

\section{Discussion}

Recently, several cases of illnesses were suspected of being caused by nanoparticles exposure and were reported in medical literatures. Two cases were reported in Germany and one in China, South Africa and Canada [47-52]. Although these cases have never been confirmed to be caused by inhalation of nanoparticles, there is increasing public, governmental, and scientific concerns on the potential adverse health effects of nanoparticle exposure. In order to avoid the selection bias of cross-sectional and short-term follow-up studies [53, 54], a long-term longitudinal study design with five repeated examinations in an interval of four years was designed to explore the potential adverse health effects of workers handling engineered nanoparticles. The researchers emphasize that this study was not to try to answer "What are the health effects of nanoparticles?" Instead, the study sought to answer "What are the potential adverse health effects among workers handling nanomaterials who are potentially exposed to nanoparticles?"

The findings of this four-year panel study showed that antioxidant enzymes, SOD, and GPx, were significantly associated with nanomaterial handling. However, cardiovascular dysfunction, lung damages, inflammation, oxidative damages, neurobehavioral and genotoxic markers were not found to be associated with nanomaterials handling in this panel study. Several possibilities can be used to explain the negative findings of health impact in

Table 5 Generalized estimating equation analysis of $\geq 2$ repeated measurements of cardiovascular disease markers over 4 years

\begin{tabular}{|c|c|c|c|c|c|c|c|c|c|}
\hline \multirow[b]{3}{*}{ Variables $^{a}$} & \multicolumn{2}{|c|}{ Control } & \multicolumn{2}{|c|}{ Risk Level 1} & \multicolumn{2}{|c|}{ Risk Level 2} & \multicolumn{3}{|c|}{ GEE analysis coefficient B (SE) } \\
\hline & \multirow[b]{2}{*}{$N$} & \multirow[b]{2}{*}{ Mean \pm SD } & \multirow[b]{2}{*}{$N$} & \multirow[b]{2}{*}{ Mean $\pm S D$} & \multirow[b]{2}{*}{ N } & \multirow[b]{2}{*}{ Mean $\pm S D$} & \multicolumn{2}{|c|}{ Model 1} & \multirow{2}{*}{$\begin{array}{c}\text { Model } 2 \\
\text { RL1*Time }\end{array}$} \\
\hline & & & & & & & RL1*Time & RL2*Time & \\
\hline Fibrinogen (mg/dL) 1st & 35 & $263.56 \pm 45.17$ & 53 & $274.17 \pm 49.14$ & 46 & $286.77 \pm 67.83$ & $-3.420(3.136)$ & $-6.490(3.494)$ & $-3.248(1.746)$ \\
\hline Fibrinogen (mg/dL) 2nd & 75 & $249.49 \pm 43.28$ & 80 & $263.08 \pm 56.28$ & 67 & $261.08 \pm 51.43$ & & & \\
\hline Fibrinogen (mg/dL) 3rd & 72 & $219.14 \pm 42.73$ & 74 & $217.83 \pm 42.33$ & 72 & $225.28 \pm 43.47$ & & & \\
\hline Fibrinogen $(\mathrm{mg} / \mathrm{dL}) 4$ th & 75 & $213.5 \pm 54.15$ & 67 & $225.52 \pm 57.38$ & 63 & $218.51 \pm 62.15$ & & & \\
\hline Fibrinogen (mg/dL) 5th & 64 & $221.2 \pm 54.41$ & 52 & $225.57 \pm 61.48$ & 57 & $219.59 \pm 66.26$ & & & \\
\hline VCAM (ng/mL) 1st & 34 & $612.9 \pm 151.48$ & 53 & $590.67 \pm 178.76$ & 46 & $642.79 \pm 203.85$ & $-24.322(10.469) \star$ & $-33.739(10.115) \star \star$ & $-16.788(4.937)$ \\
\hline VCAM $(\mathrm{ng} / \mathrm{mL}) 2 \mathrm{nd}$ & 75 & $462.74 \pm 160.74$ & 81 & $516.37 \pm 166.86$ & 68 & $514.06 \pm 164.67$ & & & \\
\hline VCAM $(\mathrm{ng} / \mathrm{mL}) 3 \mathrm{rd}$ & 71 & $429.03 \pm 127.83$ & 72 & $426.52 \pm 139.26$ & 71 & $393.77 \pm 131.75$ & & & \\
\hline VCAM $(\mathrm{ng} / \mathrm{mL})$ 4th & 79 & $372.81 \pm 101.62$ & 68 & $359.93 \pm 100.05$ & 64 & $375.79 \pm 109.01$ & & & \\
\hline VCAM $(\mathrm{ng} / \mathrm{mL}) 5$ th & 64 & $565.41 \pm 106.74$ & 52 & $563.16 \pm 103.29$ & 56 & $569.2 \pm 126.19$ & & & \\
\hline ICAM (ng/mL) 1st & 35 & $505.42 \pm 137.68$ & 53 & $463.57 \pm 136.36$ & 46 & $498.75 \pm 150.27$ & $2.674(8.871)$ & $-0.024(8.996)$ & $0.243(4.505)$ \\
\hline ICAM (ng/mL) 2nd & 75 & $443.67 \pm 202.98$ & 81 & $525.75 \pm 197.94$ & 67 & $538.21 \pm 218.68$ & & & \\
\hline ICAM (ng/mL) 3rd & 73 & $421.23 \pm 124.21$ & 73 & $449.52 \pm 137.34$ & 71 & $435.78 \pm 138.17$ & & & \\
\hline ICAM (ng/mL) 4th & 79 & $331.37 \pm 128.31$ & 68 & $338.84 \pm 119.72$ & 65 & $321.29 \pm 132.41$ & & & \\
\hline ICAM (ng/mL) 5th & 64 & $222.29 \pm 64.61$ & 52 & $196.7 \pm 54.22$ & 56 & $240.85 \pm 83.54$ & & & \\
\hline $\mathrm{hsCRP}(\mathrm{mg} / \mathrm{L}) 1 \mathrm{st}$ & 33 & $0.61 \pm 0.55$ & 51 & $0.72 \pm 0.6$ & 39 & $0.81 \pm 0.92$ & $-0.071(0.044)$ & $-0.038(0.055)$ & $-0.022(0.276)$ \\
\hline $\mathrm{hsCRP}$ (mg/L) 2nd & 75 & $0.7 \pm 0.77$ & 74 & $0.76 \pm 0.88$ & 64 & $0.64 \pm 0.71$ & & & \\
\hline $\mathrm{hsCRP}(\mathrm{mg} / \mathrm{L}) 3 \mathrm{rd}$ & 67 & $0.81 \pm 0.9$ & 69 & $1 \pm 1.05$ & 69 & $1.04 \pm 1.08$ & & & \\
\hline hsCRP (mg/L) 4th & 76 & $0.75 \pm 0.77$ & 60 & $0.73 \pm 0.8$ & 61 & $0.92 \pm 0.83$ & & & \\
\hline hsCRP (mg/L) 5th & 63 & $0.94 \pm 0.97$ & 46 & $1.06 \pm 1.23$ & 54 & $0.93 \pm 0.94$ & & & \\
\hline
\end{tabular}

atimes of measurements (1st: at baseline; $2 \mathrm{nd}$ : at 6 months later; 3rd: at 18 months later; 4th: at 30 months later; 5 th: at 42 months later)

$\star \star p<0.01 ; \star 0.01<p<0.05$

Analytical Model (Dependent variable: Fibrinogen) includes main effects of age, sex, smoking, chewing betel nut, and education.

Analytical Model (Dependent variable: VCAM) includes main effects of sex, and smoking.

Analytical Model (Dependent variable: ICAM) includes main effects of sex, smoking, and asthma.

Analytical Model (Dependent variable: hsCRP) includes main effects of sex, smoking, drinking, hypertension, and hyperlipidemia. 
Table 6 Generalized estimating equation analysis of $\geq 2$ repeated measurements of cardiovascular disease markers over 4 years

\begin{tabular}{|c|c|c|c|c|c|c|c|c|c|}
\hline \multirow[b]{3}{*}{ Variables $^{a}$} & \multicolumn{2}{|c|}{ Control } & \multicolumn{2}{|c|}{ Risk Level 1} & \multicolumn{2}{|c|}{ Risk Level 2} & \multicolumn{3}{|c|}{ GEE analysis coefficient B (SE) } \\
\hline & \multirow[b]{2}{*}{$N$} & \multirow[b]{2}{*}{ Mean \pm SD } & \multirow[b]{2}{*}{$N$} & \multirow[b]{2}{*}{ Mean \pm SD } & \multirow[b]{2}{*}{ N } & \multirow[b]{2}{*}{ Mean \pm SD } & \multicolumn{2}{|c|}{ Model 1} & \multirow{2}{*}{$\begin{array}{l}\text { Model } 2 \\
\text { RL1*Time }\end{array}$} \\
\hline & & & & & & & RL1*Time & RL2*Time & \\
\hline $\mathrm{IL}-6(\mathrm{pg} / \mathrm{mL}) 1 \mathrm{st}$ & 35 & $9.9 \pm 4.04$ & 53 & $9.27 \pm 3.34$ & 45 & $9.81 \pm 4.2$ & $-0.314(0.285) \star \star$ & $-0.759(0.276)$ & $-0.355(0.136) \star \star$ \\
\hline IL-6 (pg/mL) 2nd & 74 & $4.35 \pm 3.29$ & 82 & $5.45 \pm 3.83$ & 66 & $5.54 \pm 3.95$ & & & \\
\hline IL-6 $(\mathrm{pg} / \mathrm{mL}) 3 \mathrm{rd}$ & 73 & $2.79 \pm 3.03$ & 73 & $6.11 \pm 11.35$ & 72 & $3.95 \pm 8.53$ & & & \\
\hline$\| \mathrm{L}-6(\mathrm{pg} / \mathrm{mL}) 4 \mathrm{th}$ & 79 & $3.89 \pm 7.51$ & 68 & $3.51 \pm 7.77$ & 65 & $1.71 \pm 2.2$ & & & \\
\hline$\| \mathrm{L}-6(\mathrm{pg} / \mathrm{mL}) 5 \mathrm{th}$ & 46 & $3.06 \pm 2.29$ & 41 & $2.49 \pm 2.4$ & 44 & $2.04 \pm 1.61$ & & & \\
\hline IL-6 sR (ng/mL) 1st & 35 & $37.88 \pm 11.76$ & 53 & $35.64 \pm 10.1$ & 46 & $34.79 \pm 10.65$ & $0.523(0.752)$ & $0.809(0.700)$ & $0.377(0.348)$ \\
\hline IL-6 sR (ng/mL) 2nd & 75 & $46.45 \pm 11.87$ & 82 & $40.72 \pm 11.04$ & 68 & $41.14 \pm 11.87$ & & & \\
\hline IL-6 sR (ng/mL) 3rd & 73 & $33.87 \pm 13.35$ & 73 & $32.02 \pm 12$ & 72 & $33.77 \pm 12.01$ & & & \\
\hline IL-6 sR (ng/mL) 4th & 79 & $31.52 \pm 9.91$ & 68 & $31.63 \pm 8.87$ & 65 & $31.21 \pm 9.24$ & & & \\
\hline IL-6 sR (ng/mL) 5th & 64 & $48.57 \pm 10.07$ & 52 & $49.68 \pm 10.61$ & 57 & $46.72 \pm 10.2$ & & & \\
\hline MPO (ng/mL) 1st & 33 & $63.14 \pm 28.91$ & 51 & $77.26 \pm 53.71$ & 43 & $84.61 \pm 51.55$ & $-6.446(4.372)$ & $-6.877(4.660)$ & $-3.600(2.305)$ \\
\hline $\mathrm{MPO}(\mathrm{ng} / \mathrm{mL}) 2 \mathrm{nd}$ & 74 & $90.94 \pm 36.38$ & 81 & $106.97 \pm 56.87$ & 66 & $117.94 \pm 70.02$ & & & \\
\hline MPO $(\mathrm{ng} / \mathrm{mL}) 3 \mathrm{rd}$ & 73 & $88.83 \pm 47.01$ & 74 & $105.16 \pm 44.11$ & 71 & $96.79 \pm 43$ & & & \\
\hline $\mathrm{MPO}(\mathrm{ng} / \mathrm{mL}) 4 \mathrm{th}$ & 79 & $171.54 \pm 80.19$ & 68 & $166.01 \pm 79.85$ & 65 & $184.4 \pm 94.49$ & & & \\
\hline $\mathrm{MPO}(\mathrm{ng} / \mathrm{mL}) 5$ th & 63 & $164.63 \pm 96.94$ & 51 & $186.14 \pm 128.59$ & 57 & $156.37 \pm 102.1$ & & & \\
\hline Arylesterase 1st & 34 & $128.67 \pm 29.24$ & 51 & $128.32 \pm 24.02$ & 44 & $124.5 \pm 25.05$ & $-4.563(1.741) \star \star$ & $-2869(1.597)$ & $-1.531(0.789)$ \\
\hline Arylesterase 2nd & 74 & $87.33 \pm 29.67$ & 82 & $99.2 \pm 30.37$ & 67 & $96.48 \pm 28.3$ & & & \\
\hline Arylesterase 3rd & 73 & $83.38 \pm 13.19$ & 74 & $80.23 \pm 12.03$ & 72 & $81.22 \pm 12.16$ & & & \\
\hline Arylesterase 5th & 64 & $91.88 \pm 15.35$ & 52 & $95.51 \pm 20.73$ & 57 & $90.2 \pm 16.5$ & & & \\
\hline
\end{tabular}

atimes of measurements (1st: at baseline; 2 nd: at 6 months later; 3rd: at 18 months later; 4th: at 30 months later; 5 th: at 42 months later)

$\star \star p$-values interaction term $<0.01$ in the GEE model

Analytical Model (Dependent variable: IL-6) includes main effects of sex, and smoking.

Analytical Model (Dependent variable: IL-6 sR) includes main effects of age, sex, and smoking.

Analytical Model (Dependent variable: MPO) includes main effects of age, sex, smoking, drinking, arrhythmia, and hypertension

Analytical Model (Dependent variable: Arylesterase) includes main effects of sex, and smoking.

this panel study. First, misclassification of exposure may lead to the underestimation of health effects. Since the researchers did not conduct environmental and personal sampling of nanoparticles, misclassification of exposure was possible. Second, negative confounders may underestimate the health effects and lead to no difference between exposed and control groups. Since the study evaluated and controlled confounders during data analysis for each effect marker, confounding bias was unlikely to be of concern in this study though some residual confounding could not be completely excluded. Third, loss to follow-up may lead to bias if loss to follow-up is associated with both exposure (risk levels) and disease (effect markers). Since loss to follow-up was not found to be associated with exposure (risk levels) in this study, loss to follow-up bias was not possible. Fourth, negligible exposure to engineered nanoparticles may lead to negative health impacts. Based on field studies conducted by Taiwan Institute of Occupational Safety and Health (IOSH) that measured the emission of nanoparticles in different operations or processes in nanotechnology industry (Table 10), nanoparticle emission was found to be negligible in enclosed operation of coating of nanomaterials, enclosed operation of mixing or grinding of nanopigments, and wet process in synthesis or centrifuge of nanomaterials. However, limited amount of nanoparticle emissions could be detected in spray drying of nanomaterials as well as dry process in polishing, milling and grinding. Table 1 indicates that most of the studied factories used liquid suspension and wet process. But there were still some factories using powder in operations, which may lead to the emission of small amounts of nanomaterials. Environmental monitoring in some of the studied plants showed that mass concentrations, surface area, and particles count of nanoparticles measured in post-operation were only slightly higher than those in pre-operation. The low concentrations of nanoparticles may induce an increase of early reaction of antioxidant enzymes, but the concentrations of nanoparticles were not high enough to induce oxidative damages and toxic effects of other organs. Therefore, nanomaterials handling in current negligible emission scenarios may not have health impact on workers in regards to cardiopulmonary injuries and 
Table 7 Generalized estimating equation analysis of $\geq 2$ repeated measurements of cardiovascular disease markers over 4 years

\begin{tabular}{|c|c|c|c|c|c|c|c|c|c|}
\hline \multirow{3}{*}{ Variables $^{a}$} & \multicolumn{2}{|c|}{ Control } & \multicolumn{2}{|c|}{ Risk Level 1} & \multicolumn{2}{|c|}{ Risk Level 2} & \multicolumn{3}{|c|}{ GEE analysis coefficient B (SE) } \\
\hline & \multirow[b]{2}{*}{ N } & \multirow[b]{2}{*}{ Mean \pm SD } & \multirow[b]{2}{*}{ N } & \multirow[b]{2}{*}{ Mean \pm SD } & \multirow[b]{2}{*}{$N$} & \multirow[b]{2}{*}{ Mean \pm SD } & \multicolumn{2}{|c|}{ Model 1} & \multirow{2}{*}{$\begin{array}{l}\text { Model } 2 \\
\text { RL1*Time }\end{array}$} \\
\hline & & & & & & & RL1*Time & RL2*Time & \\
\hline paraoxonase (unit/ml) 1st & 34 & $1121.64 \pm 401.63$ & 51 & $1196.94 \pm 488.25$ & 44 & $1205.68 \pm 390.9$ & $-10.619(14.943)$ & $-18.242(12.892)$ & $-9.259(6.455)$ \\
\hline paraoxonase (unit/ml) 2nd & 74 & $900.4 \pm 337.11$ & 82 & $920.9 \pm 358.07$ & 67 & $936.96 \pm 312.4$ & & & \\
\hline paraoxonase (unit/ml) 3rd & 73 & $869.72 \pm 338.36$ & 74 & $951.92 \pm 298.78$ & 72 & $915.69 \pm 292.91$ & & & \\
\hline paraoxonase (unit/ml) 4th & 79 & $830.34 \pm 325.89$ & 68 & $920.14 \pm 352.64$ & 65 & $851.78 \pm 314.39$ & & & \\
\hline paraoxonase (unit/ml) 5th & 64 & $779.12 \pm 300.61$ & 52 & $872.7 \pm 341.33$ & 57 & $847.03 \pm 286.93$ & & & \\
\hline SDNN (ms) 1st & 34 & $39.09 \pm 12.02$ & 52 & $45.91 \pm 17.25$ & 45 & $43.57 \pm 15.45$ & $-1.541(0.873)$ & $-1.614(0.887)$ & $-0.817(0.444)$ \\
\hline SDNN (ms) 2nd & 75 & $39.59 \pm 16.06$ & 81 & $43.57 \pm 18.71$ & 67 & $44.33 \pm 17.26$ & & & \\
\hline SDNN (ms) 3rd & 73 & $43.64 \pm 18.82$ & 74 & $43.92 \pm 17.18$ & 71 & $44.2 \pm 18.42$ & & & \\
\hline SDNN (ms) 4th & 80 & $39.25 \pm 16.21$ & 66 & $40.1 \pm 14.12$ & 65 & $39.35 \pm 17.8$ & & & \\
\hline SDNN (ms) 5th & 62 & $39.6 \pm 16.47$ & 51 & $42.11 \pm 16.31$ & 57 & $37.65 \pm 13.69$ & & & \\
\hline RMSSD (ms) 1st & 34 & $25.07 \pm 11.06$ & 52 & $32.92 \pm 14.96$ & 46 & $31.02 \pm 15.77$ & $-1.234(0.716)$ & $-1.203(0.763)$ & $-0.596(0.383)$ \\
\hline RMSSD (ms) 2nd & 73 & $27.4 \pm 13.68$ & 81 & $31.46 \pm 18.8$ & 66 & $30.56 \pm 16.39$ & & & \\
\hline RMSSD (ms) 3rd & 72 & $29.34 \pm 14.52$ & 73 & $28.5 \pm 15.21$ & 70 & $28.84 \pm 14.93$ & & & \\
\hline RMSSD (ms) 4th & 79 & $24.13 \pm 11.42$ & 67 & $27.09 \pm 13.8$ & 64 & $24.62 \pm 12.9$ & & & \\
\hline RMSSD (ms) 5th & 65 & $23.6 \pm 10.29$ & 51 & $25.54 \pm 12.35$ & 57 & $24.88 \pm 12.31$ & & & \\
\hline $\mathrm{VLF}(\mathrm{ms} 2) 1 \mathrm{st}$ & 34 & $595.94 \pm 546.57$ & 51 & $652.99 \pm 516.35$ & 44 & $568.88 \pm 381.44$ & $-35.240(35.351)$ & $-24.379(33.075)$ & $-12.450(16.564)$ \\
\hline VLF (ms2) 2nd & 74 & $572.78 \pm 560.28$ & 79 & $655.26 \pm 636.07$ & 66 & $642.27 \pm 536.73$ & & & \\
\hline VLF (ms2) 3rd & 71 & $573.79 \pm 543.63$ & 70 & $546.25 \pm 448.1$ & 65 & $561.91 \pm 539.93$ & & & \\
\hline VLF (ms2) 4th & 78 & $510.31 \pm 421.48$ & 67 & $646.18 \pm 601.7$ & 61 & $520.77 \pm 495.83$ & & & \\
\hline VLF (ms2) 5th & 59 & $642.16 \pm 641.35$ & 49 & $576.27 \pm 522$ & 55 & $532.74 \pm 510.33$ & & & \\
\hline LF (ms2) 1st & 35 & $611.56 \pm 480.14$ & 52 & $694.45 \pm 613.32$ & 44 & $751.32 \pm 739$ & $-14.202(30.830)$ & $-53.531(32.371)$ & $-25.967(16.115)$ \\
\hline LF (ms2) 2nd & 73 & $447.93 \pm 386.22$ & 79 & $575.7 \pm 544.75$ & 65 & $598.24 \pm 536.82$ & & & \\
\hline LF (ms2) 3rd & 71 & $486.59 \pm 495.96$ & 73 & $554.67 \pm 533.98$ & 69 & $611.94 \pm 594.38$ & & & \\
\hline LF (ms2) 4th & 78 & $424.3 \pm 419.4$ & 66 & $522.92 \pm 448.81$ & 62 & $508.42 \pm 484.99$ & & & \\
\hline LF (ms2) 5th & 62 & $560.55 \pm 540.79$ & 50 & $657.62 \pm 554.21$ & 57 & $465.33 \pm 445.06$ & & & \\
\hline $\mathrm{HF}(\mathrm{ms} 2) 1 \mathrm{st}$ & 34 & $235.81 \pm 236.02$ & 50 & $236.19 \pm 171$ & 45 & $296.39 \pm 254.19$ & 5.993 (10.816) & 1.368 (12.301) & $0.841(6.119)$ \\
\hline HF (ms2) 2nd & 73 & $225.51 \pm 189.97$ & 77 & $259.41 \pm 226.35$ & 64 & $234.28 \pm 141.02$ & & & \\
\hline $\mathrm{HF}(\mathrm{ms} 2) 3 \mathrm{rd}$ & 70 & $230.99 \pm 202.36$ & 70 & $261.96 \pm 242.84$ & 68 & $281.85 \pm 236.15$ & & & \\
\hline $\mathrm{HF}(\mathrm{ms} 2)$ 4th & 76 & $185.35 \pm 171.39$ & 65 & $263.85 \pm 246.1$ & 63 & $253.78 \pm 271.83$ & & & \\
\hline $\mathrm{HF}(\mathrm{ms} 2)$ 5th & 62 & $193.68 \pm 165.35$ & 50 & $254.4 \pm 219.26$ & 56 & $192.98 \pm 167.61$ & & & \\
\hline LF/HF ratio $1 \mathrm{st}$ & 35 & $3.34 \pm 1.96$ & 51 & $3.04 \pm 2.57$ & 45 & $3.93 \pm 3.62$ & $0.026(0.149)$ & $-202(0.162)$ & $-0.105(0.081)$ \\
\hline LF/HF ratio 2 nd & 73 & $2.55 \pm 2.09$ & 78 & $2.69 \pm 2.46$ & 66 & $2.96 \pm 2.53$ & & & \\
\hline LF/HF ratio 3rd & 71 & $2.55 \pm 2.51$ & 73 & $2.68 \pm 2.56$ & 72 & $3.05 \pm 2.83$ & & & \\
\hline LF/HF ratio 4 th & 77 & $2.76 \pm 2.53$ & 63 & $2.35 \pm 1.79$ & 62 & $2.75 \pm 2.51$ & & & \\
\hline LF/HF ratio 5th & 61 & $3.09 \pm 2.47$ & 51 & $3.59 \pm 3.21$ & 56 & $3.23 \pm 2.74$ & & & \\
\hline
\end{tabular}

atimes of measurements (1st: at baseline; 2nd: at 6 months later; 3rd: at 18 months later; 4th: at 30 months later; 5 th: at 42 months later)

Analytical Model (Dependent variable: paraoxonase) includes main effects of sex, and smoking.

Analytical Model (Dependent variable: SDNN) includes main effects of age, sex, smoking, chewing betel nut, and education.

Analytical Model (Dependent variable: RMSSD) includes main effects of age, sex, smoking, chewing betel nut, and education.

Analytical Model (Dependent variable: VLF) includes main effects of sex, and smoking.

Analytical Model (Dependent variable: LF) includes main effects of age, sex, smoking, hyperlipidemia, and education.

Analytical Model (Dependent variable: $\mathrm{HF}$ ) includes main effects of age, sex, and smoking.

Analytical Model (Dependent variable: LF/HF ratio) includes main effects of sex, and smoking. 
Table 8 Generalized estimating equation analysis of $\geq 2$ repeated measurements of pulmonary function over 4 years

\begin{tabular}{|c|c|c|c|c|c|c|c|c|c|}
\hline \multirow{3}{*}{$\overline{\text { Variables }^{a}}$} & \multicolumn{2}{|c|}{ Control } & \multirow{2}{*}{\multicolumn{2}{|c|}{ Risk Level 1}} & \multirow{2}{*}{\multicolumn{2}{|c|}{ Risk Level 2}} & \multicolumn{3}{|c|}{ GEE analysis coefficient B(SE) } \\
\hline & \multirow[b]{2}{*}{$N$} & \multirow[b]{2}{*}{ Mean \pm SD } & & & & & \multicolumn{2}{|c|}{ Model 1} & \multirow{2}{*}{$\begin{array}{l}\text { Model } 2 \\
\text { RL1*Time }\end{array}$} \\
\hline & & & $N$ & Mean \pm SD & $N$ & Mean \pm SD & RL1*Time & RL2*Time & \\
\hline FVC (\%) 1st & 34 & $104.36 \pm 10.86$ & 53 & $106.17 \pm 13.26$ & 46 & $104.54 \pm 13.2$ & $1.164(0.071)$ & $0.468(0.715)$ & $0.269(0.356)$ \\
\hline FVC (\%) 2nd & 74 & $110.53 \pm 20.59$ & 81 & $109.51 \pm 14.51$ & 68 & $110.02 \pm 15.39$ & & & \\
\hline FVC (\%) 3rd & 73 & $106.18 \pm 14.56$ & 74 & $107.66 \pm 12.05$ & 72 & $104.48 \pm 11.86$ & & & \\
\hline FVC (\%) 4th & 77 & $108.14 \pm 17.74$ & 67 & $110.75 \pm 14.62$ & 64 & $107.49 \pm 13.07$ & & & \\
\hline FVC (\%) 5th & 65 & $106.06 \pm 15.67$ & 52 & $112.57 \pm 15.93$ & 57 & $107.94 \pm 13.34$ & & & \\
\hline FEV1.0/FVC (\%) 1st & 34 & $79.91 \pm 7.24$ & 53 & $82.23 \pm 5.5$ & 46 & $81.32 \pm 6.43$ & $-0.269(0.328)$ & $-0.084(0.332)$ & $-0.05(1.673)$ \\
\hline FEV1.0/FVC (\%)2nd & 74 & $81.43 \pm 6.54$ & 81 & $81.59 \pm 4.92$ & 68 & $81.47 \pm 5.78$ & & & \\
\hline FEV1.0/FVC (\%) 3rd & 73 & $80.49 \pm 6.1$ & 74 & $80.67 \pm 5.45$ & 72 & $82.02 \pm 5.73$ & & & \\
\hline FEV1.0/FVC (\%) 4th & 77 & $79.46 \pm 6.93$ & 67 & $79.47 \pm 5.43$ & 63 & $78.87 \pm 6.07$ & & & \\
\hline FEV1.0/FVC (\%) 5th & 65 & $80.34 \pm 6.22$ & 52 & $78.43 \pm 5.56$ & 57 & $79.57 \pm 6.01$ & & & \\
\hline MMF (\%) 1st & 34 & $83.29 \pm 27.74$ & 53 & $88.53 \pm 21.09$ & 46 & $84.69 \pm 22.79$ & $0.737(1.084)$ & $0.694(1.082)$ & $0.346(0.542)$ \\
\hline MMF (\%) 2nd & 74 & $91.41 \pm 25.02$ & 81 & $87.84 \pm 20.43$ & 68 & $91.6 \pm 27.21$ & & & \\
\hline MMF (\%) 3rd & 73 & $82.13 \pm 22.35$ & 74 & $83.45 \pm 19.68$ & 72 & $86.99 \pm 21.13$ & & & \\
\hline MMF (\%) 4th & 78 & $81.63 \pm 20.93$ & 67 & $81.69 \pm 20.38$ & 64 & $79.1 \pm 22.4$ & & & \\
\hline MMF (\%) 5th & 65 & $81.28 \pm 21.85$ & 52 & $79.7 \pm 21.17$ & 57 & $80.38 \pm 22.13$ & & & \\
\hline PEFR (\%) 1st & 34 & $99.03 \pm 19.79$ & 53 & $102.48 \pm 13.82$ & 46 & $99.93 \pm 14.53$ & $0.663(0.889)$ & $0.746(0.962)$ & $0.413(0.480)$ \\
\hline PEFR (\%) 2nd & 74 & $101.91 \pm 17.9$ & 81 & $102.86 \pm 18.56$ & 68 & $105.52 \pm 20.51$ & & & \\
\hline PEFR (\%) 3rd & 73 & $98.48 \pm 15.69$ & 74 & $105.89 \pm 14.9$ & 72 & $103.23 \pm 16.68$ & & & \\
\hline PEFR (\%) 4th & 78 & $94.96 \pm 17.81$ & 67 & $104.76 \pm 12.95$ & 64 & $99.08 \pm 18.79$ & & & \\
\hline PEFR (\%) 5th & 65 & $98.62 \pm 15.35$ & 52 & $104.23 \pm 13.73$ & 57 & $102 \pm 20.13$ & & & \\
\hline
\end{tabular}

a. times of measurements (1st: at baseline; 2 nd: at 6 months later; 3rd: at 18 months later; 4th: at 30 months later; 5 th: at 42 months later)

Analytical Model (Dependent variable: FVC) includes main effects of sex, smoking, allergic dermatitis, and education.

Analytical Model (Dependent variable: FEV1.0/FVC (\%)) includes main effects of age, sex, smoking, asthma, and arrhythmia.

Analytical Model (Dependent variable: MMF (\%)) includes main effects of sex, smoking, chronic bronchitis, and angina pectoris.

Analytical Model (Dependent variable: PEFR (\%)) includes main effects of sex, smoking, chronic bronchitis, and angina pectoris.

oxidative damages, except for the increase of antioxidant enzymes that physiologically responds to negligible nanoparticle exposure. However, further study is needed to investigate whether exposure to higher concentration of nanoparticles or working longer in nanotechnology, other than operations or processes that were studied in this study, can cause health effects.

Increase of antioxidant enzymes (SOD and GPX) was the only finding in this study. This finding was inconsistent with our previous cross-sectional study [20] and 6month follow-up study [19]. In the cross-sectional study, we found that the antioxidant enzyme SOD in risk level 1 and risk level 2 workers was significantly $(\mathrm{p}<0.05)$ lower than in control workers. A significantly decreasing gradient with risk levels was found for SOD (control > risk level $1>$ risk level 2). Another antioxidant, glutathione peroxidase (GPX), was significantly lower only in risk level 1 workers than in the control workers. The cardiovascular markers, fibrinogen and ICAM, were significantly higher in risk level 2 workers than in controls, and a significant increasing gradient with risk levels was found for these two cardiovascular markers. Another cardiovascular marker, interleukin-6, was significantly increased among risk level 1 workers, but not risk level 2 workers [20]. While in the 6-month follow-up study, the researchers found that changes of the antioxidant enzymes (decrease of superoxide dismutase and glutathione peroxidase), cardiovascular markers (increase of vascular cell adhesion molecule, decrease of paraoxonase), the small airway damage marker (decrease of Clara cell protein 16), and lung function parameters (decrease of MMF, PEFR and FEF25\%) were significantly associated with nanomaterial-handling during the 6month follow-up period [19].

This discrepancy may be attributed to selection bias in cohort studies where the selection of study population into the cohort is somehow related to the probability of the outcome studied. An increased hazard in the time period after inclusion in the cohort may be caused by the study population with the outcome studied may be more prone to be recruited as members of the cohort $[53,54]$. Selection bias of this type is likely to be more 
Table 9 Generalized estimating equation analysis of $\geq 2$ repeated measurements of pulmonary function over 4 years

\begin{tabular}{|c|c|c|c|c|c|c|c|c|c|}
\hline \multirow{3}{*}{$\overline{\text { Variables }^{a}}$} & \multirow{2}{*}{\multicolumn{2}{|c|}{ Control }} & \multirow{2}{*}{\multicolumn{2}{|c|}{ Risk Level 1}} & \multirow{2}{*}{\multicolumn{2}{|c|}{ Risk Level 2}} & \multicolumn{3}{|c|}{ GEE analysis coefficient B (SE) } \\
\hline & & & & & & & \multicolumn{2}{|c|}{ Model 1} & \multirow{2}{*}{$\begin{array}{l}\text { Model } 2 \\
\text { RL1*Time }\end{array}$} \\
\hline & $N$ & Mean \pm SD & $N$ & Mean \pm SD & $N$ & Mean \pm SD & RL1*Time & RL2*Time & \\
\hline FEF25(\%) 1st & 34 & $98.9 \pm 24.62$ & 53 & $103.5 \pm 17.42$ & 46 & $97.52 \pm 17.93$ & $1.269(0.966)$ & $1.337(1.091)$ & $0.717(0.548)$ \\
\hline FEF25(\%) 2nd & 74 & $103.91 \pm 22.57$ & 81 & $104.78 \pm 18.88$ & 68 & $105.08 \pm 25.18$ & & & \\
\hline FEF25(\%) 3rd & 73 & $96.11 \pm 18.66$ & 74 & $105.06 \pm 18.88$ & 72 & $101.56 \pm 20.59$ & & & \\
\hline FEF25(\%) 4th & 78 & $95.01 \pm 19.87$ & 67 & $104.29 \pm 17.03$ & 64 & $97.66 \pm 20.74$ & & & \\
\hline FEF25(\%) 5th & 65 & $97.02 \pm 18.31$ & 52 & $101.71 \pm 17.88$ & 57 & $98.54 \pm 23$ & & & \\
\hline FEF50(\%) 1st & 34 & $82.84 \pm 25.1$ & 53 & $87.64 \pm 21.8$ & 46 & $85.24 \pm 21.66$ & $1.002(1.064)$ & $0.549(1.029)$ & $0.305(0.519)$ \\
\hline FEF50(\%) 2nd & 74 & $90.64 \pm 26.62$ & 81 & $88.68 \pm 20.19$ & 68 & $91.92 \pm 27.01$ & & & \\
\hline FEF50(\%) 3rd & 73 & $83.09 \pm 23.96$ & 74 & $84.68 \pm 19.41$ & 72 & $87.51 \pm 22.3$ & & & \\
\hline FEF50(\%) 4th & 78 & $81.97 \pm 21.32$ & 67 & $84.03 \pm 19.02$ & 64 & $82.5 \pm 22.02$ & & & \\
\hline FEF50(\%) 5th & 65 & $80.15 \pm 21.84$ & 52 & $82.11 \pm 21.41$ & 57 & $81.84 \pm 20.57$ & & & \\
\hline FEF75(\%) 1st & 34 & $58.53 \pm 26.86$ & 53 & $64.07 \pm 22.69$ & 46 & $60.24 \pm 25.06$ & $-0.16(1.230)$ & $-0.04(1.224)$ & $-0.048(0.613)$ \\
\hline FEF75(\%) 2nd & 74 & $64.91 \pm 22.94$ & 81 & $61.12 \pm 20.74$ & 68 & $66.05 \pm 25.75$ & & & \\
\hline FEF75(\%) 3rd & 73 & $56.92 \pm 19.78$ & 74 & $57.15 \pm 21.07$ & 72 & $60.79 \pm 17.83$ & & & \\
\hline FEF75(\%) 4th & 77 & $56.91 \pm 22.45$ & 67 & $53.76 \pm 17.94$ & 64 & $51.62 \pm 19.02$ & & & \\
\hline FEF75(\%) 5th & 65 & $58.15 \pm 24.74$ & 50 & $51.74 \pm 15.32$ & 57 & $55.31 \pm 23.12$ & & & \\
\hline
\end{tabular}

${ }^{a}$ times of measurements (1st: at baseline; $2 \mathrm{nd}$ : at 6 months later; 3rd: at 18 months later; 4 th: at 30 months later; 5 th: at 42 months later) Analytical Model (Dependent variable: FEF25 (\%)) includes main effects of sex, smoking, chronic bronchitis, and angina pectoris.

Analytical Model (Dependent variable: FEF50 (\%)) includes main effects of sex, smoking, and chronic bronchitis.

Analytical Model (Dependent variable: FEF75 (\%)) includes main effects of age, sex, smoking, asthma, and arrhythmia.

pronounced shortly after inclusion into the cohort. Cross-sectional study and short-term (e.g.6-month) follow-up study would have a high probability of suffering from this type of selection bias and result in positive health impacts. After some time period, the health problems leading to inclusion in the cohort could be resolved. Removal of observation time and events occurring shortly after inclusion in the cohort could reduce the impact of selection bias [53, 54]. In order to avoid such type of selection bias, this panel study used a long-term longitudinal study design with five repeated examinations in an interval of four years. The study concluded that nanomaterials handling in current negligible emission scenarios may not have health impact on the workers regarding cardiopulmonary injuries and oxidative damages, except for the increase of antioxidant enzymes physical response to the negligible emission of nanomaterials.

There are several limitations in this panel study. First, the small size of the study population limits its generalizability. The heterogeneity of the nanomaterials made it difficult to find a sufficiently large group of workers exposed to the same nanomaterial and to present potential health effects of any one nanomaterial. Second, misclassification of exposure by control banding was possible. Such random misclassification did not lead to systematic bias; random

Table 10 Emission of nanomaterials in different operations or processes in nanotechnology industry

\begin{tabular}{lc}
\hline Operation & Emission of Nanomaterials \\
\hline Coating of nanomaterials (Enclosed) & Negligible \\
Mixing/grinding of nanopigments (Enclosed) & Negligible \\
Synthesis of nanomaterials (Wet process) & Negligible \\
Centrifuge of nanomaterials (wet process) & Negligible \\
Spray drying of nanomaterials & Yes \\
Welding & Yes \\
Polishing & Yes \\
Milling & Yes \\
Grinding & Yes \\
Foundry lndustry & Yes
\end{tabular}


misclassification is biased toward the null value, which underestimates the true risk. Third, chronic effect markers should be used to evaluate the chronic, long-term health hazard of nanomaterials handling workers. Fourth, failure to set up the model for concurrent exposure to varied nanomaterials in order to understand the interaction among different nanomaterials. Finally, there is the risk to underestimate the effect of NPs exposure if workers with high exposures would not participate in the follow-up exams. This risk is low because we collected workers' specimens during a series of annual health examinations, which provides the absence pattern of workers. However, we cannot fully exclude a healthy worker effect in which workers quit the job because of health issues before they started to participate in the study.

In order to prevent the hazards of handling nanomaterials, the introduction of strict preventive measures, such as local ventilation and personal protective equipment, are still the only way to prevent the risk of occupational disease in workers handling nanomaterials. Although negligible nanoparticle exposure was detected in the enclosed operation and the wet process of nanomaterials handling, limited amount of nanoparticles can still be detected in spray drying of nanomaterials as well as dry process in polishing, milling and grinding. Respiratory protection in addition to local ventilation should be implemented in nanotechnology in order to reduce nanoparticles exposure and to prevent nanoparticles-induced illnesses.

\section{Conclusions}

An increasing gradient with risk levels of superoxide dismutase (SOD) and increase of glutathione peroxidase in RL 1 were found for nanomaterials handling workers in comparison to the controls. Industrial hygiene surveys and environmental emission surveys indicated that exposure concentrations of nanoparticles was dramatically reduced due to wet processes and liquid suspensions used in the workplace. Although nanomaterial handling in current low emission scenarios may not have 4-year health impact on the workers, long-term health effects of more than 5 years for nanomaterial used still need research.

\footnotetext{
Abbreviation

CC16: Clara cell protein; NO: Nitric oxide; NF-kB: Nuclear factor-kappaB; 8OHdG: 8-hydroxydeoxyguanosine; SOD: Superoxide dismutase; GPx: Glutathione peroxidase; VCAM: Vascular cell adhesion molecule; ICAM: Intercellular adhesion molecule; hsCRP: High sensitive C-reactive protein; MPO: Myeloperoxidase; IL-6: Interleukin-6; IL-6sR: Interleukin-6 soluble receptor; HRV: Heart rate variability; SDNN: Standard deviation of all normal to normal R-R intervals; RMSSD: The root mean square of successive differences between adjacent normal cycles; VLF: Very low frequency; LF: Low frequency; HF: High frequency; LF/HF: Low frequency/high frequency ratio; FVC: Forced vital capacity; FEV1: Forced expiratory volume at 1 second; MMF: Maximal mid-expiratory flow; PEFR: Peak expiratory flow rate; FEF25\%: Forced expiratory flow at 25\%; FEF50\%: Forced expiratory flow at 50\%; FEF75\%: Forced expiratory flow at $75 \%$
}

\section{Acknowledgements}

The authors thank Dr. C.J. Tsai at National Chiao-Tung University, Taiwan and Dr. P.J. Tsai at Chen-Kung University, Taiwan for their assistance in the exposure assessments of nanoparticles in the workplace. The authors thank Dr. C.J. Chen and Dr. MH Lin in Institute of Labor, Occupational Safety, and Health, Ministry of Labor for their assistance and cooperation.

\section{Authors' contributions}

LA, TC, SL, and HL carried out the assays of health effect markers. WT performed the statistical analysis and draft the manuscript. SH conceived of the study, and participated in its design and coordination. All authors read and approved the final manuscript.

\section{Funding}

This study was partly supported by the National Health Research Institutes of Taiwan (grants 98A1-EOSP03-014, 99A1-EOSP03-014, 00A1-EOSP03-014, and 01A1-EOSP03-014) and the Institute of Occupational Safety and Health, Taiwan (grants IOSH98-M323, IOSH99-M323, IOSH100-M323, and IOSH101M323). The funder had no role in study design, data collection and analysis, decision to publish, or preparation of the manuscript.

\section{Availability of data and materials}

The datasets generated during and/or analysed during the current study are available from the corresponding author on reasonable request.

\section{Ethics approval and consent to participate}

This study has been approved by the institutional review board of the National Health Research Institutes of Taiwan (No. EC0971003-E).

\section{Consent for publication}

This study has not been published previously and is not under consideration for publication elsewhere. The corresponding author is responsible on behalf of all co-authors for all aspects of the research and writing process, and take final responsibility for this paper.

\section{Competing interests}

The authors declare that they have no competing interests.

\section{Author details}

${ }^{1}$ National Institute of Environmental Health Sciences, National Health Research Institutes, Miaoli County, Taiwan. ${ }^{2}$ Institute of Environmental and Occupational Health Sciences, National Yang Ming University, Taipei, Taiwan. ${ }^{3}$ Department of Chemistry, Fu Jen Catholic University, Taipei, Taiwan.

${ }^{4}$ Institute of Labor, Occupational Safety, and Health, Ministry of Labor, Taipei, Taiwan. ${ }^{5}$ Division of occupational medicine, Division of fanily medicine, Department of Family and Community Medicine, Tri-Service General Hospital, National Defense Medical Center, Taipei, Taiwan.

Received: 27 June 2019 Accepted: 6 November 2019

Published online: 09 December 2019

\section{References}

1. Bekker C, Brouwer DH, Tielemans E, Pronk A. Industrial production and professional application of manufactured nanomaterials-enabled end products in Dutch industries: potential for exposure. Ann Occup Hyg. 2013; 57(3):314-27.

2. Borm PJ, Robbins D, Haubold S, Kuhlbusch T, Fissan H, Donaldson K, Schins $\mathrm{R}$, Stone $\mathrm{V}$, Kreyling W, Lademann J, et al. The potential risks of nanomaterials: a review carried out for ECETOC. Part Fibre Toxicol. 2006:3:11.

3. Oberdorster G, Oberdorster E, Oberdorster J. Nanotoxicology: an emerging discipline evolving from studies of ultrafine particles. Environ Health Perspect. 2005:113(7):823-39.

4. Hesterberg TW, Bunn WB 3rd, Chase GR, Valberg PA, Slavin TJ, Lapin CA, Hart GA. A critical assessment of studies on the carcinogenic potential of diesel exhaust. Crit Rev Toxicol. 2006;36(9):727-76.

5. Hesterberg TW, Long CM, Bunn WB, Sax SN, Lapin CA, Valberg PA. Non-cancer health effects of diesel exhaust: a critical assessment of recent human and animal toxicological literature. Crit Rev Toxicol. 2009;39(3):195-227.

6. Hesterberg TW, Long CM, Lapin CA, Hamade AK, Valberg PA. Diesel exhaust particulate (DEP) and nanoparticle exposures: what do DEP human clinical 
studies tell us about potential human health hazards of nanoparticles? Inhal Toxicol. 2010;22(8):679-94.

7. Hubbs AF, Mercer RR, Benkovic SA, Harkema J, Sriram K, Schwegler-Berry D, Goravanahally MP, Nurkiewicz TR, Castranova V, Sargent LM. Nanotoxicology-a pathologist's perspective. Toxicol Pathol. 2011;39(2):301-24.

8. Stern ST, McNeil SE. Nanotechnology safety concerns revisited. Toxicol Sci. 2008;101(1):4-21.

9. DHHS, US. NIOSH. Current Intelligence Bulletin 65: Occupational Exposure to Carbon Nanotubes and Nanofibers. National Institute for Occupational Safety and Health.

10. Schubauer-Berigan MK, Dahm MM, Erdely A, Beard JD, Birch ME, Evans DE, Fernback JE, Mercer RR, Bertke SJ, Eye TJP, et al. Association of pulmonary, cardiovascular, and hematologic metrics with carbon nanotube and nanofiber exposure among US workers: a cross-sectional study. Part Fibre Toxicol. 2018;15(1):22.

11. Beard JD, Erdely A, Dahm MM, de Perio MA, Birch ME, Evans DE, Fernback JE, Eye T, Kodali V, RRJEi M. Carbon nanotube and nanofiber exposure and sputum and blood biomarkers of early effect among US workers. Environ Int. 2018;116:214-28.

12. Brouwer DH. Control banding approaches for nanomaterials. Ann Occup Hyg. 2012;56(5):506-14.

13. Riediker M, Schubauer-Berigan MK, Brouwer DH, Nelissen I, Koppen G, Frijns E, Clark KA, Hoeck J, Liou SH, Ho SF, et al. A road map toward a globally harmonized approach for occupational health surveillance and epidemiology in nanomaterial workers. J Occup Environ Med. 2012;54(10):1214-23.

14. Brouwer D. Exposure to manufactured nanoparticles in different workplaces. Toxicology. 2010;269(2-3):120-7.

15. Brouwer D, Berges M, Virji MA, Fransman W, Bello D, Hodson L, Gabriel S, Tielemans $E$. Harmonization of measurement strategies for exposure to manufactured nano-objects; report of a workshop. Ann Occup Hyg. 2012; 56(1):1-9.

16. Dahm MM, Evans DE, Schubauer-Berigan MK, Birch ME, Fernback JE. Occupational exposure assessment in carbon nanotube and nanofiber primary and secondary manufacturers. Ann Occup Hyg. 2012;56(5):542-56.

17. Kuhlbusch TA, Asbach C, Fissan H, Göhler D, Stintz MJP. Nanoparticle exposure at nanotechnology workplaces: a review. Part Fibre Toxicol. 2011; 8(1):22.

18. Paik SY, Zalk DM, Swuste P. Application of a pilot control banding tool for risk level assessment and control of nanoparticle exposures. Ann Occup Hyg. 2008:52(6):419-28.

19. Liao H-Y, Chung Y-T, Lai C-H, Wang S-L, Chiang H-C, Li L-A, Tsou T-C, Li W-F, Lee H-L, Wu W-TJN. Six-month follow-up study of health markers of nanomaterials among workers handling engineered nanomaterials. Nanotoxicology. 2014;8(sup 1):100

20. Liou S-H, Tsou T-C, Wang S-L, Li L-A, Chiang H-C, Li W-F, Lin P-P, Lai C-H, Lee $\mathrm{H}-\mathrm{L}$, Lin MH, Hsu JH. Epidemiological study of health hazards among workers handling engineered nanomaterials. J Nanopart Res. 2012;14(8):878.

21. Liao H-Y, Chung Y-T, Wu W-T, Huang Y-F, Lai C-H, Lin M-H, Hsu J-H, Chen CJ, Shih T-S, S-HJ L. Industrial Hygiene Survey of Engineered Nanomaterials Handling Factories in Taiwan Nanotechnology Industry. 中華職業醫學雜誌. 2013;20(3):139-56.

22. Ostiguy C, Lapointe G. Ménard Luk. Studies and Research Projects. Montréal: Health Effects of Nanoparticles. IRSST. Report R-589; 2008. p. 114.

23. Wichmann H-E, Spix C, Tuch T, Wölke G, Peters A, Heinrich J, Kreyling WG, JJRr H. Daily mortality and fine and ultrafine particles in Erfurt, Germany part I: role of particle number and particle mass. J Expo Sci Environ Epidemiol. 2000;98:5-86 discussion 87-94.

24. Frampton MWJI. Does inhalation of ultrafine particles cause pulmonary vasular effects in humans? Inhal Toxicol. 2007;19(sup1):75-9.

25. Araujo JA, Barajas B, Kleinman M, Wang X, Bennett BJ, Gong KW, Navab M, Harkema J, Sioutas C, Lusis AJ. Ambient particulate pollutants in the ultrafine range promote early atherosclerosis and systemic oxidative stress. Circ Res. 2008;102(5):589-96.

26. Araujo JA, AEJP N. Particulate matter and atherosclerosis: role of particle size, composition and oxidative stress. Part Fibre Toxicol. 2009;6(1):24.

27. Peters A, Breitner S, Cyrys J, Stölzel M, Pitz M, Wölke G, Heinrich J, Kreyling W, Küchenhoff $\mathrm{H}$, Wichmann $\mathrm{H}-\mathrm{E}$. The influence of improved air quality on mortality risks in Erfurt, Germany. Res Rep Health Eff Inst. 2009;137:5-77.

28. Quan C, Sun Q, Lippmann M, Chen L-C. Comparative effects of inhaled diesel exhaust and ambient fine particles on inflammation, atherosclerosis, and vascular dysfunction. Inhal Toxicol. 2010;22(9):738-53.
29. Bernard A, Gonzalez-Lorenzo J, Siles E, Trujillano G, Lauwerys R. Early decrease of serum Clara cell protein in silica-exposed workers. Eur Respir J. 1994;7(11):1932-7.

30. Njemini R, Lambert M, Demanet C, Mets T. Elevated serum heat-shock protein 70 levels in patients with acute infection: use of an optimized enzyme-linked immunosorbent assay. Scand J Immunol. 2003:58(6):664-9.

31. Silkoff PE, Hunt JF. ATS workshop proceedings: exhaled nitric oxide and nitric oxide oxidative metabolism in exhaled breath condensate: executive summary. Am J Respir Crit Care Med. 2006;173(7):811.

32. Taylor D, Pijnenburg M, Smith A, Jongste JD. Exhaled nitric oxide measurements: clinical application and interpretation. Thorax. 2006;61(9): $817-27$.

33. Tsou T-C, Yeh S-C, Tsai F-Y, Lin H-J, Cheng T-J, Chao H-R, Tai L-A. Zinc oxide particles induce inflammatory responses in vascular endothelial cells via NFKB signaling. J Hazard Mater. 2010;183(1-3):182-8.

34. Zelko IN, Mariani TJ, Folz RJ. Superoxide dismutase multigene family: a comparison of the CuZn-SOD (SOD1), Mn-SOD (SOD2), and EC-SOD (SOD3) gene structures, evolution, and expression. Medicine. 2002;33(3):337-49.

35. Delfino RJ, Staimer N, Tjoa T, Polidori A, Arhami M, Gillen DL, Kleinman MT, Vaziri ND, Longhurst J, Zaldivar F. Circulating biomarkers of inflammation, antioxidant activity, and platelet activation are associated with primary combustion aerosols in subjects with coronary artery disease. Environ Health Perspect. 2008;1 16(7):898-906.

36. Chen H-I, Liou S-H, Ho S-F, Wu K-Y, Sun C-W, Chen M-F, Cheng L-C, Shih T$\mathrm{S}$, Loh C-H. Oxidative DNA damage estimated by plasma 8hydroxydeoxyguanosine (8-OHdG): influence of 4, 4'-methylenebis (2chloroaniline) exposure and smoking. J Occup Health. 2007;49(5):389-98.

37. Wang C-J, Yang N-H, Chang C-C, Liou S-H, Lee H-L. Rapid and simple onestep membrane extraction for the determination of 8-hydroxy-2'deoxyguanosine in human plasma by a combination of on-line solid phase extraction and LC-MS/MS. J Chromatogr B. 2011;879(30):3538-43.

38. Wang C-J, Yang N-H, Liou S-H, Lee H-LTT. Fast quantification of the exhaled breath condensate of oxidative stress 8-iso-prostaglandin F2a using on-line solid-phase extraction coupled with liquid chromatography/electrospray ionization mass spectrometry. Talanta. 2010;82(4):1434-8.

39. Marui N, Offermann MK, Swerlick R, Kunsch C, Rosen CA, Ahmad M, Alexander RW, Medford RM. Vascular cell adhesion molecule-1 (VCAM-1) gene transcription and expression are regulated through an antioxidant-sensitive mechanism in human vascular endothelial cells. J Clin Invest. 1993;92(4):1866-74.

40. Li W-F, Sun C-W, Cheng T-J, Chang K-H, Chen C-J, Wang S-LJT. Risk of carotid atherosclerosis is associated with low serum paraoxonase (PON1) activity among arsenic exposed residents in Southwestern Taiwan. Toxicol Appl Pharmacol. 2009;236(2):246-53.

41. Ridker PM. C-reactive protein and the prediction of cardiovascular events among those at intermediate risk: moving an inflammatory hypothesis toward consensus. J Am Coll Cardiol. 2007;49(21):2129-38.

42. Timonen KL, Vanninen E, De Hartog J, Ibald-Mulli A, Brunekreef B, Gold DR, Heinrich J, Hoek G, Lanki T, Peters A, et al. Effects of ultrafine and fine particulate and gaseous air pollution on cardiac autonomic control in subjects with coronary artery disease: the ULTRA study. J Expo Sci Environ Epidemiol. 2006;16(4):332.

43. Karlsson $\mathrm{HL}$. The comet assay in nanotoxicology research. Anal Bioanal Chem. 2010;398(2):651-66.

44. Wu P-A, Loh C-H, Hsieh L-L, Liu T-Y, Chen C-J, Liou SH. Clastogenic effect for cigarette smoking but not areca quid chewing as measured by micronuclei in exfoliated buccal mucosal cells. Mutat Res. 2004;562(1-2):27-38.

45. Qaseem A, Wilt TJ, Weinberger SE, Hanania NA, Criner G, van der Molen T, Marciniuk DD, Denberg T, Schünemann H, Wedzicha W. Diagnosis and management of stable chronic obstructive pulmonary disease: a clinical practice guideline update from the American College of Physicians, American College of Chest Physicians, American Thoracic Society, and European Respiratory Society. Ann Intern Med. 2011;155(3):179-91.

46. Tsai S-Y, Chen J-D, Chao WY, Wang JD. Neurobehavioral effects of occupational exposure to low-level organic solvents among Taiwanese workers in paint factories. Environ Res. 1997;73(1-2):146-55.

47. Wolinsky H. Nanoregulation: a recent scare involving nanotech products reveals that the technology is not yet properly regulated. EMBO Rep. 2006; 7(9):858-61.

48. Pauluhn J, Hahn A, Spielmann HJI. Assessment of early acute lung injury in rats exposed to aerosols of consumer products: attempt to disentangle the "Magic Nano" conundrum. Inhal Toxicol. 2008;20(14):1245-62. 
49. Song Y, Li X, Du X. Exposure to nanoparticles is related to pleural effusion, pulmonary fibrosis and granuloma. Eur Respir J. 2009;34(3):559-67.

50. Theegarten D, Boukercha S, Philippou S, Anhenn O. Submesothelial deposition of carbon nanoparticles after toner exposition: case report. Diagn Pathol. 2010;5(1):77.

51. Phillips Jl, Green FY, Davies JC, Murray J. Pulmonary and systemic toxicity following exposure to nickel nanoparticles. Am J Ind Med. 2010;53(8):763-7.

52. Journeay WS, Goldman RH. Occupational handling of nickel nanoparticles: a case report. Am J Ind Med. 2014;57(9):1073-6.

53. Törner A, Duberg A-S, Dickman P, Svensson A. A proposed method to adjust for selection bias in cohort studies. Am J Epidemiol. 2010;171(5):602-8.

54. Törner A, Dickman P, Duberg A-S, Kristinsson S, Landgren O, Björkholm M, Svensson A. A method to visualize and adjust for selection bias in prevalent cohort studies. Am J Epidemiol. 2011;174(8):969-76.

\section{Publisher's Note}

Springer Nature remains neutral with regard to jurisdictional claims in published maps and institutional affiliations.

- fast, convenient online submission

- thorough peer review by experienced researchers in your field

- rapid publication on acceptance

- support for research data, including large and complex data types

- gold Open Access which fosters wider collaboration and increased citations

- maximum visibility for your research: over $100 \mathrm{M}$ website views per year

At $\mathrm{BMC}$, research is always in progress. 